\title{
Analysis and Finite Element Approximation of a Nonlinear Stationary Stokes Problem Arising in Glaciology
}

\author{
Guillaume Jouvet $^{1,2}$ and Jacques Rappaz ${ }^{2}$ \\ ${ }^{1}$ Department of Mathematics and Computer Science, Free University of Berlin, 14195 Berlin, Germany \\ ${ }^{2}$ Chair of Numerical Analysis and Simulation, EPFL, 1015 Lausanne, Switzerland
}

Correspondence should be addressed to Guillaume Jouvet, guillaume.jouvet@fu-berlin.de

Received 13 September 2011; Accepted 25 October 2011

Academic Editor: Michele Benzi

Copyright (C) 2011 G. Jouvet and J. Rappaz. This is an open access article distributed under the Creative Commons Attribution License, which permits unrestricted use, distribution, and reproduction in any medium, provided the original work is properly cited.

The aim of this paper is to study a nonlinear stationary Stokes problem with mixed boundary conditions that describes the ice velocity and pressure fields of grounded glaciers under Glen's flow law. Using convex analysis arguments, we prove the existence and the uniqueness of a weak solution. A finite element method is applied with approximation spaces that satisfy the inf-sup condition, and a priori error estimates are established by using a quasinorm technique. Several algorithms (including Newton's method) are proposed to solve the nonlinearity of the Stokes problem and are proved to be convergent. Our results are supported by numerical convergence studies.

\section{Introduction}

In this paper we consider a model problem that is commonly used by glaciologists to compute the motion of glaciers. Ice is assumed to be an incompressible non-Newtonian fluid governed by Glen's law [1]. Glen's law and the mass momentum equation lead to a nonlinear stationary Stokes problem with a strain-dependent viscosity.

Glacier models based on Glen's law have already been studied by several authors. However, all of them have considered a simplified model, called first-order approximation [2]. This model is obtained by rewriting the Stokes equations into a dimensionless form and by dropping all terms of order $\mathcal{O}\left(\epsilon^{2}\right)$, where $\epsilon$ is the typical aspect ratio of glaciers. This simplification results into a nonlinear elliptic problem for the horizontal velocity field, the vertical component, and the pressure field being determined a posteriori. Colinge and Rappaz first demonstrated the well-posedness of this problem and proved the convergence of 
the finite element approximation with piecewise linear continuous functions in [3]. Inspired by the work of Baranger/Najib [4] and Barrett/Liu [5] on non-Newtonian problems, a priori and a posteriori error estimates were obtained later in [6-8].

Unlike the first-order approximation, the original Stokes model which is considered in this paper is a saddle point problem for the velocity and the pressure fields. We prove the existence and the uniqueness of a weak solution using an equivalent minimisation problem and an inf-sup stability condition. Next, we establish a priori estimates for a finite element approximation using a quasinorm technique [5]. Eventually, we investigate several successive approximation algorithms to solve the system nonlinearity. In particular, we upgrade by using Newton's method the fixed point algorithm, given in [3] and proved to be convergent in $[6,9]$.

Boundary conditions describe the basal sliding phenomena that can significantly influence the glacier ice flows. In [3,6-8], the first order approximation model was coupled to a Dirichlet condition. However, this approach requires the basal velocity distribution which is unknown. To overcome this difficulty, several sliding laws-including a Coulomb-type law-were considered in [10]. In our model, we use a sliding law that results in a nonlinear Dirichlet-Robin boundary condition.

This paper is organised as follows: the physical model is presented in Section 2. We prove the well-posedness of the weak problem in Section 3. In Section 4, we apply a finite element method and establish a priori error estimates. Successive approximation algorithms to solve the system nonlinearity are proposed and proved to be convergent in Section 5. In Section 6, convergence studies are performed to support the results of Sections 4 and 5.

\section{The Model}

Let us suppose that ice occupies the domain $\Omega \subset \mathbb{R}^{d}$, with $d=2$ or 3 . Ice can be considered as an incompressible non-Newtonian fluid with negligible inertial effects [11]. It follows that the velocity $\mathbf{u}$ and the pressure $p$ of ice solve the stationary nonlinear Stokes problem in $\Omega$ :

$$
\begin{gathered}
-2 \operatorname{div}(\mu \varepsilon(\mathbf{u}))+\nabla p=\mathbf{f}, \\
\operatorname{div}(\mathbf{u})=0,
\end{gathered}
$$

where $\varepsilon(\mathbf{u})=(1 / 2)\left(\nabla \mathbf{u}+\nabla \mathbf{u}^{T}\right)$ denotes the rate of strain tensor, $\mu$ the viscosity of ice, and $\mathbf{f}$ the gravity force. Here above, the viscosity $\mu$ depends on $|\varepsilon(\mathbf{u})|:=\sqrt{\varepsilon(\mathbf{u}): \varepsilon(\mathbf{u})}$ and is defined by the regularised Glen's flow law [11]. More precisely, for a given velocity field $\mathbf{u}$, the viscosity $\mu$ satisfies the following nonlinear equation:

$$
\frac{1}{2 \mu}=A\left(\tau_{0}^{n-1}+(\sqrt{2} \mu s)^{n-1}\right)
$$

where $s=|\varepsilon(\mathbf{u})|, A$ is a positive parameter, $n \geq 1$ is Glen's exponent, and $\tau_{0}>0$ is a small regularization parameter which prevents infinite viscosity for zero strain $\left(\tau_{0}=0\right.$ in the original Glen's law [1]). When $n=1$, then the viscosity $\mu$ is constant and (2.1) correspond 
to the classical linear Stokes problem related to a Newtonian fluid. In the framework of glaciology, $n$ is often taken equal to 3; see [12].

Let us set the boundary conditions for the system of (2.1). Three mechanical circumstances may occur at the boundary of a glacier: (i) no force applies on the ice-air interface; (ii) ice slides on the bedrock-ice interface; (iii) ice is stuck to the bedrock-ice interface. The boundary of $\Omega$ is thus split into three parts: $\Gamma_{N}, \Gamma_{R}$, and $\Gamma_{D}$, referring to circumstances (i), (ii), and (iii), respectively. We assume throughout that $\Omega$ is bounded, its boundaries $\Gamma_{N}$ and $\Gamma_{R}$, are $\mathcal{C}^{1}$ and $\Gamma_{D} \neq \emptyset$. We consider the free surface condition:

$$
2 \mu \varepsilon(\mathbf{u}) \cdot \mathbf{n}-p \mathbf{n}=\mathbf{0}, \quad \text { on } \Gamma_{N},
$$

where $\mathbf{n}$ is the unit outward normal vector along the boundary of the domain $\Omega$. We apply the nonlinear sliding condition [10, 13, 14]:

$$
\mathbf{u} \cdot \mathbf{n}=0, \quad(2 \mu \varepsilon(\mathbf{u}) \cdot \mathbf{n}) \cdot \mathbf{t}_{i}=-\alpha \mathbf{u} \cdot \mathbf{t}_{i}, \quad i=1, d-1 \quad \text { on } \Gamma_{R},
$$

where $\left\{\mathbf{t}_{i}\right\}_{i=1, d-1}$ are the orthogonal vectors tangent to the boundary $\Gamma_{R}$, that is, $\mathbf{t}_{1}$ when $d=2$ and $\mathbf{t}_{1}, \mathbf{t}_{2}$ when $d=3$. Here above, $\alpha=\alpha(|\mathbf{u}|)$ is the sliding coefficient that is given by

$$
\alpha(t)=c\left(t+t_{0}\right)^{1 / n-1}
$$

where $t=|\mathbf{u}|$ is the Euclidean norm of $\mathbf{u}, n$ is Glen's exponent, $c$ is a positive parameter, and $t_{0}>0$ is a small parameter which prevents infinite $\alpha$ for zero velocity. The no-sliding condition writes

$$
\mathbf{u}=\mathbf{0}, \quad \text { on } \Gamma_{D}
$$

Note that the conditions applied on boundaries $\Gamma_{N}, \Gamma_{R}$, and $\Gamma_{D}$ are Neumann, RobinDirichlet, and Dirichlet conditions, respectively. When $n=1$ (Newtonian flow) and $\Gamma_{R}=\emptyset$, the problem (2.1) with boundary conditions (2.3), (2.6) has already been widely studied; see, for instance, [15-17].

\section{Existence and Uniqueness}

In this section, we prove that there exists a unique weak solution to problem (2.1) with mixed boundary conditions (2.3), (2.4), and (2.6). Pressure is first eliminated from the system by restricting the velocity space to divergence-free fields. Afterwards, the reduced problem is transformed into a minimisation problem. Following [3, 8], its well-posedness is proved by using convex analysis arguments. The existence and the uniqueness of the pressure field are ensured by an inf-sup condition. We now state in the next lemma several properties of the function $\mu$ that will often be used in Sections 3, 4 and 5 . 
Lemma 3.1. For all $s \in \mathbb{R}_{+}$, there exists a unique $\mu=\mu(s) \in \mathbb{R}_{+}$satisfying (2.2). The function $s \rightarrow \mu(s)$ is $\mathcal{C}^{\infty}(0,+\infty)$ and decreasing. There exist $D_{1}, D_{2}, D_{3}, D_{4}>0$ such that:

$$
\begin{gathered}
\frac{D_{1}}{(1+s+t)^{1-1 / n}}(s-t) \leq s \mu(s)-t \mu(t) \leq \frac{D_{2}}{(1+s+t)^{1-(1 / n)}}(s-t), \quad \forall s \geq t \geq 0, \\
\frac{D_{1}}{(1+s)^{1-1 / n}} \leq \mu(s) \leq \frac{D_{2}}{(1+s)^{1-1 / n}}, \quad \forall s \geq 0, \\
\frac{1}{n} \mu(s) \leq \mu(s)+s \mu^{\prime}(s), \quad \forall s>0, \\
(\mu(|\xi|) \xi-\mu(|\eta|) \eta):(\xi-\eta) \geq D_{3}(1+|\xi|+|\xi-\eta|)^{(1 / n-1)}|\xi-\eta|^{2}, \quad \forall \xi, \eta \in \mathbb{R}^{d \times d}, \\
|\mu(|\xi|) \xi-\mu(|\eta|) \eta| \leq D_{4}(1+|\xi|+|\xi-\eta|)^{(1 / n-1)}|\xi-\eta|, \quad \forall \xi, \eta \in \mathbb{R}^{d \times d} .
\end{gathered}
$$

Proof. The properties of $\mu$ and inequalities (3.1) and (3.2) can be easily deduced from Lemmas 1 and 2 of [7]. Inequality (3.3) is obtained by differentiating (2.2) with respect to s. Inequalities (3.4) and (3.5) result from inequality (3.1), Lemma 2.1 in [5] and inequality $(1 / 2)(|\xi|+|\eta|) \leq|\xi|+|\xi-\eta| \leq 2(|\xi|+|\eta|)$. Details are given in [12].

Let us notice that property (3.1) was introduced by Barrett and Liu (see [5]) in order to obtain a priori error estimates of a similar problem to the one treated in this paper. Define the Banach spaces:

$$
V:=\left\{\mathbf{v} \in\left[W^{1, r}(\Omega)\right]^{d}, \mathbf{v}=\mathbf{0} \text { on } \Gamma_{D}, \mathbf{v} \cdot \mathbf{n}=0 \text { on } \Gamma_{R}\right\}, \quad Q:=L^{r^{\prime}}(\Omega),
$$

where

$$
r:=1+\frac{1}{n}, \quad r^{\prime}:=n+1
$$

are conjugate exponents and $n$ is Glen's exponent. By using (3.2), we have $\mu(s) s \leq C s^{r-1}$ for all $s>0$. Then, if $\mathbf{u} \in V$, we have $\mu(|\varepsilon(\mathbf{u})|) \varepsilon(\mathbf{u}) \in\left[L^{r^{\prime}}(\Omega)\right]^{d \times d}$. By using the trace inequality $\|\mathbf{v}\|_{L^{r}\left(\Gamma_{R}\right)} \leq\|\mathbf{v}\|_{W^{1-1 / r, r}\left(\Gamma_{R}\right)} \leq C\|\mathbf{v}\|_{W^{1, r}(\Omega)}$ for all $\mathbf{v} \in\left[W^{1, r}(\Omega)\right]^{d}$, see [19, page 197], we obtain $\left(\mathbf{u} \cdot \mathbf{t}_{i}\right) \in L^{r}\left(\Gamma_{R}\right), i=1, d-1$. Similarly, we can show $\alpha(|\mathbf{u}|)\left(\mathbf{u} \cdot \mathbf{t}_{i}\right) \in L^{r^{\prime}}\left(\Gamma_{R}\right), i=1, d-1$. Owing to Hölder's inequality, the mixed formulation of problem (2.1) with boundary conditions (2.3), (2.4), and (2.6) that consists of finding $(\mathbf{u}, p) \in V \times Q$ such that

$$
\begin{aligned}
& 2 \int_{\Omega} \mu(|\varepsilon(\mathbf{u})|) \varepsilon(\mathbf{u}): \varepsilon(\mathbf{v}) d V+\sum_{i=1, d-1} \int_{\Gamma_{R}} \alpha(|\mathbf{u}|)\left(\mathbf{u} \cdot \mathbf{t}_{i}\right)\left(\mathbf{v} \cdot \mathbf{t}_{i}\right) d S, \\
& -\int_{\Omega} p \operatorname{div}(\mathbf{v}) d V+\int_{\Omega} q \operatorname{div}(\mathbf{u}) d V=\int_{\Omega} \mathbf{g} \cdot \mathbf{v} d V, \quad \forall(\mathbf{v}, q) \in V \times Q
\end{aligned}
$$

is meaningful. 
Remark 3.2. If $\Gamma_{N}=\emptyset$, pressure $p$ in (3.8) is defined up to a constant. In that case, $Q=L^{r^{\prime}}$ is replaced by $Q=L_{0}^{r^{\prime}}=\left\{q \in L^{r^{\prime}}, \int_{\Omega} q d V=0\right\}$. Moreover, if $n=1$, then $r=2$ and $\mu$ is constant and if $\Gamma_{R}=\emptyset$, then the (linear) problem (3.8) is well posed; see, for instance, [15-17].

The next lemma states the equivalence of norms $\||\varepsilon(\cdot)|\|_{L^{r}}$ and $\|\cdot\|_{W^{1, r}}$ on space $V$.

Lemma 3.3 (Korn's inequality). If $\Gamma_{D} \neq \emptyset$ and if $1<\gamma<\infty$, then there exists a constant $C>0$ such that

$$
\|\mathbf{v}\|_{W^{1, r}} \leq C\|\| \varepsilon(\mathbf{v}) \mid \|_{L^{r}},
$$

for all $\mathbf{v} \in W^{1, \gamma}(\Omega)$ such that $\mathbf{v}=\mathbf{0}$ on $\Gamma_{D}$.

Proof. We apply Corollary 4.1 in [18] ( $F$ being the identity matrix) and Lemma 3.1 page 40 in [16].

We consider the divergence-free velocity space:

$$
V_{\mathrm{div}}:=\left\{\mathbf{v} \in\left[W^{1, r}(\Omega)\right]^{d}, \operatorname{div}(\mathbf{v})=0, \mathbf{v}=\mathbf{0} \text { on } \Gamma_{D}, \mathbf{v} \cdot \mathbf{n}=0 \text { on } \Gamma_{R}\right\} .
$$

In $V_{\mathrm{div}}$, the pressure field $p$ vanishes of the variational formulation (3.8). The reduced formulation consists then of finding $\mathbf{u} \in V_{\mathrm{div}}$ such that

$$
2 \int_{\Omega} \mu(|\varepsilon(\mathbf{u})|) \varepsilon(\mathbf{u}): \varepsilon(\mathbf{v}) d V+\sum_{i=1, d-1} \int_{\Gamma_{R}} \alpha(|\mathbf{u}|)\left(\mathbf{u} \cdot \mathbf{t}_{i}\right)\left(\mathbf{v} \cdot \mathbf{t}_{i}\right) d S=\int_{\Omega} \mathbf{g} \cdot \mathbf{v} d V, \quad \forall \mathbf{v} \in V_{\mathrm{div}}
$$

To transform problem (3.11) into a minimisation problem, we introduce the functional

$$
J(\mathbf{u}):=\int_{\Omega} M(|\varepsilon(\mathbf{u})|) d V+\frac{1}{2} \int_{\Gamma_{R}} N(|\mathbf{u}|) d S-\int_{\Omega} \mathbf{u} \cdot \mathbf{f} d V,
$$

where

$$
M(x):=\int_{0}^{x} s \mu(s) d s, \quad N(x):=\int_{0}^{x} t \alpha(t) d t
$$

The functional $J$ is Gâteaux differentiable, and its first derivative $D J$, at point $\mathbf{u} \in V_{\mathrm{div}}$, in direction $\mathbf{v} \in V_{\text {div }}$, is given by

$$
\langle D J(\mathbf{u}), \mathbf{v}\rangle=2 \int_{\Omega} \mu(|\varepsilon(\mathbf{u})|) \varepsilon(\mathbf{u}): \varepsilon(\mathbf{v}) d V+\sum_{i=1, d-1} \int_{\Gamma_{R}} \alpha(|\mathbf{u}|)\left(\mathbf{u} \cdot \mathbf{t}_{i}\right)\left(\mathbf{v} \cdot \mathbf{t}_{i}\right) d S-\int_{\Omega} \mathbf{g} \cdot \mathbf{v} d V
$$

Clearly, any minimiser of $J$ in $V_{\text {div }}$ satisfies (3.11). We now establish several lemmas that allow us to prove the existence and the uniqueness of this minimiser in Theorem 3.8. We show 
the continuity of $J$ in Lemma 3.5, the strict convexity of $J$ in Lemma 3.6, and the coercivity (in the sense of (3.18)) of $J$ in Lemma 3.7. The continuity of $J$ requires the following result (Lemma 4 in [3])

Lemma 3.4. Let $O$ be a measurable set of $\mathbb{R}^{d}$ and $f, g \in L^{r}(O)$, then one has the following inequality:

$$
\left.\int_{O}|| f\right|^{r}-|g|^{r}\left|d V \leq r\||f|+|g|\|_{L^{r}(O)}^{r-1}\|f-g\|_{L^{r}(O)} .\right.
$$

Lemma 3.5. The functional $J$ is $\|\cdot\|_{W^{1, r} \text {-continuous. }}$

Proof. By using (3.2), (2.5), and $1-1 / n=2-r$, we have, for all $\mathbf{u}, \mathbf{v} \in V_{\mathrm{div}}$

$$
\begin{aligned}
|M(|\varepsilon(\mathbf{u})|)-M(|\varepsilon(\mathbf{v})|)| & =\left|\int_{|\varepsilon(\mathbf{v})|}^{|\varepsilon(\mathbf{u})|} s \mu(s) d s\right| \leq\left|D_{1} \int_{|\varepsilon(\mathbf{v})|}^{|\varepsilon(\mathbf{u})|} s^{r-1} d s\right|=D_{1}\left|\frac{|\varepsilon(\mathbf{u})|^{r}-|\varepsilon(\mathbf{v})|^{r}}{r}\right|, \\
|N(|\mathbf{u}|)-N(|\mathbf{v}|)| & =\left|\int_{|\mathbf{v}|}^{|\mathbf{u}|} t \alpha(t) d t\right| \leq\left|c \int_{|\mathbf{v}|}^{|\mathbf{u}|} t^{r-1} d t\right|=c\left|\frac{|\mathbf{u}|^{r}-|\mathbf{v}|^{r}}{r}\right| .
\end{aligned}
$$

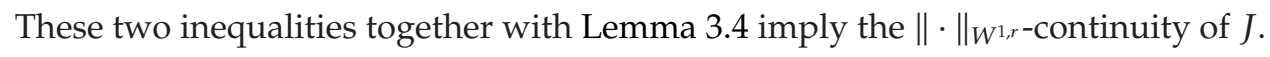

Lemma 3.6. The functional $J$ is strictly convex on $V$.

Proof. Clearly, $M^{\prime}(s)=s \mu(s)$ and $M^{\prime \prime}(s)=s \mu^{\prime}(s)+\mu(s)$. From (3.3), we have $M^{\prime \prime}(s)=s \mu^{\prime}(s)+$ $\mu(s) \geq(1 / n) \mu(s)>0$ if $s>0$, and then $M$ is strictly convex. Since $M$ is an increasing function, $M(|\cdot|)$ is strictly convex. In the same way, we can show that $N(|\cdot|)$ is strictly convex by using (2.5). Let $\mathbf{u}, \mathbf{v} \in V_{\text {div }}$ satisfying $\mathbf{u} \neq \mathbf{v}$ and $\theta \in(0,1)$. From Korn's inequality (Lemma 3.3), we have $\varepsilon(\mathbf{u}) \neq \varepsilon(\mathbf{v})$ in $L^{r}$. As a consequence,

$$
\int_{\Omega} M(|\theta \varepsilon(\mathbf{u})+(1-\theta) \varepsilon(\mathbf{v})|) d V<\theta \int_{\Omega} M(|\varepsilon(\mathbf{u})|) d V+(1-\theta) \int_{\Omega} M(|\varepsilon(\mathbf{v})|) d V
$$

The strict convexity of $J$ follows from the previous inequality and the convexity of $N(|\cdot|)$.

Since $J$ is convex, $\mathbf{u} \in V_{\text {div }}$ satisfies (3.11) if and only if $J(\mathbf{u}) \leq J(\mathbf{v}), \forall \mathbf{v} \in V_{\text {div }}$.

Lemma 3.7. There exist two constants $D_{1}, D_{2}>0$ such that, for all $\mathbf{u} \in V$,

$$
J(\mathbf{u}) \geq D_{1}\|\mathbf{u}\|_{W^{1, r}}^{r}-D_{2}
$$

Proof. Let $\mathbf{u} \in V$. From (3.2) and $1-1 / n=2-r$, there exists $C_{0}>0$ such that

$$
\begin{aligned}
M(|\varepsilon(\mathbf{u})|) & \geq \int_{0}^{|\varepsilon(\mathbf{u})|} \frac{C_{0} s}{(1+s)^{2-r}} d s \\
& =\int_{0}^{|\varepsilon(\mathbf{u})|^{2} / 2} \frac{C_{0}}{(1+\sqrt{2 t})^{2-r}} d t \geq \frac{|\varepsilon(\mathbf{u})|^{2}}{2} C_{0}(1+|\varepsilon(\mathbf{u})|)^{r-2} .
\end{aligned}
$$


As a consequence, there exist two constants $C_{1}, C_{2}>0$ such that $M(|\varepsilon(\mathbf{u})|) \geq C_{1}(1+|\varepsilon(\mathbf{u})|)^{r}-$ $C_{2}$. By using Korn's inequality (Lemma 3.3), there exists $C_{3}>0$ such that

$$
\int_{\Omega} M(|\varepsilon(\mathbf{u})|) d V \geq C_{3}\|\mathbf{u}\|_{W^{1, r}}^{r}-C_{2} \int_{\Omega} d V
$$

From Young's inequality, we have, for all $\delta>0$,

$$
\int_{\Omega}|\mathbf{u} \cdot \mathbf{f}| d V \leq \int_{\Omega}\left(\frac{1}{r^{\prime} \delta^{r^{\prime}}}|\mathbf{f}|^{r^{\prime}}+\frac{\delta^{r}}{r}|\mathbf{u}|^{r}\right) d V=\frac{C_{4}}{\delta^{r^{\prime}}} \int_{\Omega} d V+C_{5} \delta^{r}\|\mathbf{u}\|_{L^{r}}^{r}
$$

where $C_{4}, C_{5}>0$. We set $\delta$ small enough such that $C_{3}-\delta^{r} C_{5}>0$. From inequalities (3.20), (3.21), and $N \geq 0$, we obtain

$$
\begin{aligned}
J(\mathbf{u}) & =\int_{\Omega}(M(|\varepsilon(\mathbf{u})|)-\mathbf{u} \cdot \mathbf{f}) d V+\int_{\Gamma_{R}} \frac{1}{2} N(|\mathbf{u}|) d S \\
& \geq C_{3}\|\mathbf{u}\|_{W^{1, r}}^{r}-C_{2} \int_{\Omega} d V-\frac{C_{4}}{\delta^{r^{\prime}}} \int_{\Omega} d V-C_{5} \delta^{r}\|\mathbf{u}\|_{W^{1, r},}^{r}
\end{aligned}
$$

which is exactly (3.18) with $D_{1}:=C_{3}-C_{5} \delta^{r}$ and $D_{2}:=\left(C_{2}+C_{4} / \delta^{r^{\prime}}\right) \int_{\Omega} d V$.

Theorem 3.8. There exists a unique $\mathbf{u} \in V_{\mathrm{div}}$ such that $J(\mathbf{u})=\inf \left\{J(\mathbf{v}) ; \mathbf{v} \in V_{\mathrm{div}}\right\}$. Moreover, $\mathbf{u}$ is the unique solution of (3.11).

Proof. Clearly, there exists $\overline{\mathbf{u}} \in V_{\text {div }}$ such that $J(\overline{\mathbf{u}})<+\infty$. Lemma 3.7 ensures the existence of $\bar{m}=\inf \left\{J(\mathbf{v}) ; \mathbf{v} \in V_{\text {div }}\right\}$. Let $\left\{\mathbf{u}_{v}\right\}$ be a sequence of $V_{\text {div }}$ such that $\lim _{v \rightarrow \infty} J\left(\mathbf{u}_{v}\right)=\bar{m}$. There exists an integer $K$ such that, for all $v>K$, we have $\bar{m}+1>J\left(\mathbf{u}_{v}\right)$. Owing to Lemma 3.7, the sequence $\left\{\mathbf{u}_{v}\right\}$ is bounded in $V_{\text {div }}$. Since $V_{\text {div }}$ is a closed subspace of $V, V_{\text {div }}$ is reflexive. Consequently, there exist $\mathbf{u} \in V_{\text {div }}$ and a subsequence of $\left\{\mathbf{u}_{v}\right\}$ (still denoted $\left\{\mathbf{u}_{v}\right\}$ ) that converges weakly to $\mathbf{u}$ in $V_{\text {div }}$. By Lemmas 3.5 and 3.6, $J$ is weakly lower semicontinuous; see, for instance, Corollary III.8 in [19] page 38. Then, we have

$$
\bar{m}=\liminf _{v \rightarrow+\infty} J\left(\mathbf{u}_{v}\right) \geq J(\mathbf{u}) \geq \bar{m},
$$

and $J$ possesses at least one minimum $\mathbf{u} \in V_{\text {div }}$. Since $J$ is strictly convex (Lemma 3.6), this minimum is unique. Moreover, $\mathbf{u}$ is the unique solution of (3.11).

Spaces $V$ and $Q$ are required to satisfy the inf-sup condition, see $[5,20]$, to ensure the existence and the uniqueness of $p \in Q$ such that $(\mathbf{u}, p)$ satisfies the mixed formulation (3.8). The inf-sup condition is proved in $[15,21]$ when $\Gamma_{D}=\partial \Omega$ (or, equivalently, $\Gamma_{N}=\Gamma_{R}=\emptyset$ ). 
By following the proof of Proposition 5.3.2 in [22], we can easily generalise this result when $\Gamma_{R} \cup \Gamma_{N} \neq \emptyset$; see details in [12].

Lemma 3.9. Spaces $V$ and $Q$ satisfy the inf-sup condition; that is, there exists $C>0$ such that

$$
C<\inf _{q \in Q} \sup _{\mathbf{v} \in V} \frac{\int_{\Omega} q \operatorname{div}(\mathbf{v}) d V}{\|q\|_{L^{\prime}}\|\mathbf{v}\|_{W^{1, r}}} .
$$

Theorem 3.10. There exists a unique couple $(\mathbf{u}, p) \in(V, Q)$ satisfying (3.8).

Proof. Although the result is a straightforward application of Theorem 2.1 in [20] together with Theorem 3.8 and Lemma 3.9, we give all the arguments of the proof. Let $A: V \rightarrow V^{\prime}$ and $B: V \rightarrow Q^{\prime}$ be the operators defined by

$$
\begin{aligned}
& \langle A \mathbf{u}, \mathbf{v}\rangle=2 \int_{\Omega} \mu(|\varepsilon(\mathbf{u})|) \varepsilon(\mathbf{u}): \varepsilon(\mathbf{v}) d V+\sum_{i=1, d-1} \int_{\Gamma_{R}} \alpha(|\mathbf{u}|)\left(\mathbf{u} \cdot \mathbf{t}_{i}\right)\left(\mathbf{v} \cdot \mathbf{t}_{i}\right) d S, \quad \forall v \in V, \\
& \langle B \mathbf{u}, q\rangle=\int_{\Omega} q \operatorname{div} \mathbf{u} d V, \quad \forall q \in Q
\end{aligned}
$$

where $V^{\prime}$ and $Q^{\prime}$ are dual to $V$ and $Q$, respectively. From Theorem 3.8, there exists a unique $\mathbf{u} \in \operatorname{ker} B$ such that $\langle A \mathbf{u}-\mathbf{f}, \mathbf{v}\rangle=0$ for all $\mathbf{v} \in \operatorname{ker} B$, which means that $A \mathbf{u}-\mathbf{f} \in(\operatorname{ker} B)^{\perp}$. Owing to the inf-sup condition (4.1), the operator $B: V \rightarrow Q^{\prime}$ is surjective, $\operatorname{ker} B^{T}=\emptyset$, and $\mathcal{R}\left(B^{T}\right)$ is closed; see Lemma A.40 in [15]. As a consequence, $A \mathbf{u}-\mathbf{f} \in(\operatorname{ker} B)^{\perp}=\overline{\mathcal{R}\left(B^{T}\right)}=\mathcal{R}\left(B^{T}\right)$ and there exists $p \in Q$ such that $A \mathbf{u}-\mathbf{f}=B^{T} p$. Since $\operatorname{ker} B^{T}=\emptyset$, the pressure $p$ is necessarily unique. Eventually, there exists a unique couple $(\mathbf{u}, p) \in V \times Q$ satisfying

$$
\begin{gathered}
A \mathbf{u}-B^{T} p=\mathbf{f} \\
B \mathbf{u}=0
\end{gathered}
$$

or equivalently (3.8).

\section{Finite Element Approximation and A Priori Estimates}

We assume that $\Omega$ is a convex polygonal or polyhedral domain and $\tau_{h}$ is a regular mesh of $\bar{\Omega}$ parametrized by $h$, the highest diameter of the elements of $\tau_{h}$. We say that $V_{h} \subset V$ and $Q_{h} \subset Q$, some finite-dimensional approximation spaces on $\tau_{h}$ of $V$ and $Q$, satisfy the inf-sup condition if, for all $\kappa \in(1, \infty)$, there exists a constant $C_{h}>0$ such that

$$
C_{h}<\inf _{q_{h} \in Q_{h}} \sup _{\mathbf{v}_{h} \in V_{h}} \frac{\int_{\Omega} q_{h} \operatorname{div}\left(\mathbf{v}_{h}\right) d V}{\left\|q_{h}\right\|_{L^{k^{\prime}}}\left\|\mathbf{v}_{h}\right\|_{W^{1, \kappa}}} .
$$


The discrete problem is obtained by replacing the spaces $V$ and $Q$ by $V_{h}$ and $Q_{h}$, respectively. It consists of finding $\left(\mathbf{u}_{h}, p_{h}\right) \in\left(V_{h}, Q_{h}\right)$ such that

$$
\begin{aligned}
& 2 \int_{\Omega} \mu\left(\left|\varepsilon\left(\mathbf{u}_{h}\right)\right|\right) \varepsilon\left(\mathbf{u}_{h}\right): \varepsilon\left(\mathbf{v}_{h}\right) d V+\sum_{i=1, d-1} \int_{\Gamma_{R}} \alpha\left(\left|\mathbf{u}_{h}\right|\right)\left(\mathbf{u}_{h} \cdot \mathbf{t}_{i}\right)\left(\mathbf{v}_{h} \cdot \mathbf{t}_{i}\right) d S \\
& -\int_{\Omega} p_{h} \operatorname{div} \mathbf{v}_{h} d V+\int_{\Omega} q_{h} \operatorname{div} \mathbf{u}_{h} d V=\int_{\Omega} \mathbf{f} \cdot \mathbf{v}_{h} d V, \quad \forall\left(\mathbf{v}_{h}, q_{h}\right) \in\left(V_{h}, Q_{h}\right) .
\end{aligned}
$$

The discrete similar space to $V_{\mathrm{div}}$ is

$$
V_{\mathrm{div}, h}=\left\{\mathbf{v}_{h} \in V_{h} ; \int_{\Omega} q_{h} \operatorname{div}\left(\mathbf{v}_{h}\right) d V=0 ; \forall q_{h} \in Q_{h}\right\}
$$

Note that $V_{\text {div, } h}$ is not necessarily included in $V_{\text {div }}$. The discrete reduced problem consists of finding $\mathbf{u}_{h} \in V_{\mathrm{div}, h}$ such that

$$
\begin{aligned}
2 \int_{\Omega} \mu\left(\left|\varepsilon\left(\mathbf{u}_{h}\right)\right|\right) \varepsilon\left(\mathbf{u}_{h}\right): \varepsilon\left(\mathbf{v}_{h}\right) d V+\sum_{i=1, d-1} \int_{\Gamma_{R}} \alpha\left(\left|\mathbf{u}_{h}\right|\right)\left(\mathbf{u}_{h} \cdot \mathbf{t}_{i}\right)\left(\mathbf{v}_{h} \cdot \mathbf{t}_{i}\right) d S \\
=\int_{\Omega} \mathbf{g} \cdot \mathbf{v}_{h} d V, \quad \forall \mathbf{v}_{h} \in V_{\mathrm{div}, h} .
\end{aligned}
$$

Since $V_{\text {div, }} h$ is a closed subspace of $V$, Theorem 3.8 and the proof can be rewritten by replacing

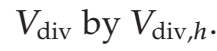

Theorem 4.1. There exists a unique $\mathbf{u}_{h} \in V_{\mathrm{div}, h}$ such that $J\left(\mathbf{u}_{h}\right)=\inf \left\{J\left(\mathbf{v}_{h}\right) ; \mathbf{v}_{h} \in V_{\mathrm{div}, h}\right\}$. Moreover, $\mathbf{u}_{h}$ is the unique solution of (4.4).

Remark 4.2. By setting $\mathbf{v}_{h}=\mathbf{u}_{h}$ in (4.4) and by using inequality (3.2), (2.5), and Korn's inequality (Lemma 3.3), we can show that the solution $\mathbf{u}_{h}$ of problem (4.4) satisfies

$$
\left\|\mathbf{u}_{h}\right\|_{W^{1, r}} \leq C\|\mathbf{f}\|_{L^{r^{\prime}}}
$$

where $C>0$ does not depend on $\mathbf{u}_{h}$.

From Theorem 4.1 and the inf-sup condition (4.1), we can rewrite Theorem 3.10 and its proof for the discrete mixed problem.

Theorem 4.3. If $V_{h}$ and $Q_{h}$ satisfy the inf-sup condition (4.1), then there exists a unique couple $\left(\mathbf{u}_{h}, p_{h}\right) \in\left(V_{h}, Q_{h}\right)$ satisfying (4.2).

Remark 4.4. The spaces $\left[\mathbb{P}_{1} / \text { Bulle }\right]^{d}-\mathbb{P}_{1}$ and $\left[\mathbb{P}_{2}\right]^{d}-\mathbb{P}_{1}$ are two examples that satisfy the inf-sup condition (4.1) while $\mathbb{P}_{1}-\mathbb{P}_{1}$ does not satisfy (4.1); see [15].

The error analysis that follows is partly inspired from [5, 7]. We give a priori estimates for the numerical approximation of the stationary Stokes problem in Theorem 4.9. 
For the sake of simplicity, we suppose $\Gamma_{R}=\emptyset$; that is, the boundary Robin-Dirichlet condition is not considered; see also Remark 4.11. The nonlinearity of problem (3.8) is treated by introducing (in Lemma 4.5) a quasi-norm that depends on the solution; see [5]. The orthogonality of the error (Lemma 4.6) together with properties (3.4) and (3.5) of the function $\mu$ allow quasi-norm estimates to be established in Theorem 4.7. The properties of the quasi-norm given in Lemma 4.5 allow estimates with standard norms to be proved in Theorem 4.8. Eventually, these estimates together with interpolation inequalities yield to the main Theorem 4.9 .

Lemma 4.5. Let $(\mathbf{u}, p)$ be the solution of (3.8); the application

$$
\mathbf{v} \longrightarrow\|\mathbf{v} \mid\|:=\sqrt{\int_{\Omega} \frac{|\varepsilon(\mathbf{v})|^{2}}{(1+|\varepsilon(\mathbf{u})|+|\varepsilon(\mathbf{v})|)^{2-r}} d V}
$$

is a quasi-norm of $V$; that is, it satisfies all properties of norms, except homogeneity. Moreover, there exists $D_{1}>0$ such that, for all $\mathbf{v} \in W^{1, r}(\Omega)$, one has

$$
\|\mathbf{v}\|_{W^{1, r}}^{2} \leq D_{1}\left[1+\|\mathbf{u}\|_{W^{1, r}}+\|\mathbf{v}\|_{W^{1, r}}\right]^{2-r}\|\mid \mathbf{v}\|^{2}
$$

and there exists $D_{2}>0$ such that, for all $\kappa \in[r, 2]$ and for all $\mathbf{v} \in W^{1, \kappa}(\Omega)$, one has

$$
\||\mathbf{v}|\|^{2} \leq D_{2}\|\mathbf{v}\|_{W^{1, \kappa}}^{\kappa} .
$$

Proof. The quasi-norm properties are shown in Lemma 3.1 in [5]. Inequalities (4.7) and (4.8) result from Korn and Hölder's inequalities; see details in [12].

By setting $\mathbf{v}=\mathbf{v}_{h} \in V_{\mathrm{div}, h}$ in (3.8) it is easy to prove the next lemma.

Lemma 4.6. Let $(\mathbf{u}, p) \in(V, Q)$ be the solution of problem (3.8) and $\mathbf{u}_{h} \in V_{\mathrm{div}, h}$ the solution of problem (4.4), then

$$
\int_{\Omega} 2\left(\mu(|\varepsilon(\mathbf{u})|) \varepsilon(\mathbf{u})-\mu\left(\left|\varepsilon\left(\mathbf{u}_{h}\right)\right|\right) \varepsilon\left(\mathbf{u}_{h}\right)\right): \varepsilon\left(\mathbf{v}_{h}\right) d V-\int_{\Omega} \operatorname{div}\left(\mathbf{v}_{h}\right)\left(p-q_{h}\right) d V=0
$$

holds for all $\left(\mathbf{v}_{h}, q_{h}\right) \in\left(V_{\text {div, } h}, Q_{h}\right)$. Moreover, if the spaces $V_{h}$ and $Q_{h}$ satisfy the inf-sup condition (4.1), then the solution $\left(\mathbf{u}_{h}, p_{h}\right)$ of (4.2) satisfies

$$
\int_{\Omega} 2\left(\mu(|\varepsilon(\mathbf{u})|) \varepsilon(\mathbf{u})-\mu\left(\left|\varepsilon\left(\mathbf{u}_{h}\right)\right|\right) \varepsilon\left(\mathbf{u}_{h}\right)\right): \varepsilon\left(\mathbf{v}_{h}\right) d V-\int_{\Omega} \operatorname{div}\left(\mathbf{v}_{h}\right)\left(p-p_{h}\right) d V=0,
$$

for all $\mathbf{v}_{h} \in V_{h}$.

Theorem 4.7. Let $(\mathbf{u}, p) \in(V, Q)$ be the solution of (3.8) and $\mathbf{u}_{h} \in V_{\mathrm{div}, h}$ the solution of (4.4). For all $\left(\mathbf{v}_{h}, q_{h}\right) \in V_{\mathrm{div}, h} \times Q_{h}$, one has

$$
\left\|\left|\mathbf{u}-\mathbf{u}_{h}\right|\right\| \leq D_{1}\left[1+\left\|\mathbf{u}_{h}\right\|_{W^{1, r}}+\left\|\mathbf{v}_{h}\right\|_{W^{1, r}}\right]^{(2-r) / 2}\left(\left\|\left|\mathbf{u}-\mathbf{v}_{h}\right|\right\|+\left\|p-q_{h}\right\|_{L^{r^{\prime}}}\right),
$$


where $D_{1}>0$. Moreover, if the spaces $V_{h}$ and $Q_{h}$ satisfy the inf-sup condition (4.1), then the solution $\left(\mathbf{u}_{h}, p_{h}\right)$ of (4.2) satisfies, for all $\kappa \in[r, 2]$ and for all $q_{h} \in Q_{h}$,

$$
\left\|p-p_{h}\right\|_{L^{\kappa^{\prime}}} \leq D_{2}\left(\left\|\mathbf{u}-\mathbf{u}_{h} \mid\right\|^{2 / \kappa^{\prime}}+\left\|p-q_{h}\right\|_{L^{\kappa^{\prime}}}\right)
$$

where $D_{2}>0$. The constants $D_{1}, D_{2}$ do not depend on $\mathbf{u}_{h}$ and $\mathbf{v}_{h}$; however, $D_{2}$ increasingly depends on $\left(C_{h}\right)^{-1}$.

Proof. By using, respectively, the definition (4.6) of the quasi-norm $\||\cdot|\|$, inequality (3.4) with $1-1 / n=2-r$, and (4.9), there exists $C_{1}>0$ such that

$$
\begin{aligned}
\left\|\mathbf{u}-\mathbf{u}_{h} \mid\right\|^{2} \leq C_{1}\left\{\int_{\Omega} 2\left(\mu(|\varepsilon(\mathbf{u})|) \varepsilon(\mathbf{u})-\mu\left(\left|\varepsilon\left(\mathbf{u}_{h}\right)\right|\right) \varepsilon\left(\mathbf{u}_{h}\right)\right): \varepsilon\left(\mathbf{u}-\mathbf{u}_{h}\right) d V\right\} \\
=C_{1}\{\underbrace{\int_{\Omega} 2\left(\mu(|\varepsilon(\mathbf{u})|) \varepsilon(\mathbf{u})-\mu\left(\left|\varepsilon\left(\mathbf{u}_{h}\right)\right|\right) \varepsilon\left(\mathbf{u}_{h}\right)\right): \varepsilon\left(\mathbf{u}-\mathbf{v}_{h}\right) d V}_{:=A_{1}} \\
+\underbrace{\int_{\Omega} \operatorname{div}\left(\mathbf{v}_{h}-\mathbf{u}_{h}\right)\left(p-q_{h}\right) d V}_{:=A_{2}}\},
\end{aligned}
$$

where $\left(\mathbf{v}_{h}, q_{h}\right) \in V_{\mathrm{div}, h} \times Q_{h}$. For the sake of simplicity, $A_{1}$ and $A_{2}$ are handled separately. By using inequality (3.5) with $1-1 / n=2-r$, there exists $C_{2}>0$ such that

$$
\begin{aligned}
\left|A_{1}\right| & \leq \int_{\Omega} 2\left|\mu(|\varepsilon(\mathbf{u})|) \varepsilon(\mathbf{u})-\mu\left(\left|\varepsilon\left(\mathbf{u}_{h}\right)\right|\right) \varepsilon\left(\mathbf{u}_{h}\right)\right|\left|\varepsilon\left(\mathbf{u}-\mathbf{v}_{h}\right)\right| d V \\
& \leq C_{2} \int_{\Omega} \frac{\left|\varepsilon\left(\mathbf{u}-\mathbf{u}_{h}\right)\right|\left|\varepsilon\left(\mathbf{u}-\mathbf{v}_{h}\right)\right|}{\left(1+|\varepsilon(\mathbf{u})|+\left|\varepsilon\left(\mathbf{u}-\mathbf{u}_{h}\right)\right|\right)^{2-r}} d V .
\end{aligned}
$$

By using the inequality (see Lemma 2.2 in [5] or (3.10) in [8]),

$$
\begin{aligned}
&(1+a+t)^{-e} t s \leq \alpha(1+a+t)^{-e} t^{2}+\alpha^{-1}(1+a+s)^{-e} s^{2}, \\
& \forall a, t, s \geq 0, \quad \forall \alpha \in(0,1], \quad e \in(0,1),
\end{aligned}
$$


with $a=|\varepsilon(\mathbf{u})|, t=\left|\varepsilon\left(\mathbf{u}-\mathbf{u}_{h}\right)\right|, s=\left|\varepsilon\left(\mathbf{u}-\mathbf{v}_{h}\right)\right|$, and $e=2-r$, we obtain, for all $\alpha \in[0,1]$,

$$
\left|A_{1}\right| \leq C_{2}\left\{\alpha\left\|\left|\mathbf{u}-\mathbf{u}_{h}\right|\right\|^{2}+\alpha^{-1}\left|\left\|\mathbf{u}-\mathbf{v}_{h} \mid\right\|^{2}\right\}\right.
$$

We now use respectively Hölder's inequality, Young's inequality, and (4.7); there exist $C_{3}, C_{4}, C_{5}>0$ such that

$$
\begin{aligned}
\left|A_{2}\right| & \leq\left\|\operatorname{div}\left(\mathbf{v}_{h}-\mathbf{u}_{h}\right)\right\|_{L^{r}}\left\|p-q_{h}\right\|_{L^{r^{\prime}}} \\
& \leq \frac{1}{2} C_{3} \beta\left\|\mathbf{v}_{h}-\mathbf{u}_{h}\right\|_{W^{1, r}}^{2}+\frac{1}{2} \beta^{-1}\left\|p-q_{h}\right\|_{L^{r^{\prime}}}^{2} \\
& \leq C_{4} \beta\left[1+\|\mathbf{u}\|_{W^{1, r}}+\left\|\mathbf{v}_{h}-\mathbf{u}_{h}\right\|_{W^{1, r}}\right]^{2-r}\left\|\mathbf{v}_{h}-\mathbf{u}_{h} \mid\right\|^{2}+\frac{1}{2} \beta^{-1}\left\|p-q_{h}\right\|_{L^{r^{\prime}}}^{2} \\
& \leq C_{5} \beta\left[1+\left\|\mathbf{u}_{h}\right\|_{W^{1, r}}+\left\|\mathbf{v}_{h}\right\|_{W^{1, r}}\right]^{2-r}\left(\left\|\mathbf{u}-\mathbf{u}_{h}\left|\left\|^{2}+\right\| \mathbf{u}-\mathbf{v}_{h}\right|\right\|^{2}\right)+\frac{1}{2} \beta^{-1}\left\|p-q_{h}\right\|_{L^{r^{\prime}}}^{2}
\end{aligned}
$$

By setting $\alpha=1 /\left(4 C_{2} C_{1}\right)$ and $\beta=1 /\left(4 C_{5} C_{1}\left[1+\left\|\mathbf{u}_{h}\right\|_{W^{1, r}}+\left\|\mathbf{v}_{h}\right\|_{W^{1, r}}\right]^{2-r}\right)$, we obtain

$$
\begin{aligned}
\left\|\left|\mathbf{u}-\mathbf{u}_{h}\right|\right\|^{2} \leq & C_{1}\left\{\left|A_{1}\right|+\left|A_{2}\right|\right\} \\
\leq & \frac{1}{2}\left\|\left|\mathbf{u}-\mathbf{u}_{h}\right|\right\|^{2}+4 C_{2}^{2} C_{1}^{2}\left\|\left|\mathbf{u}-\mathbf{v}_{h}\right|\right\|^{2} \\
& +\frac{1}{4}\left\|\mathbf{u}-\mathbf{v}_{h} \mid\right\|^{2}+2 C_{1}^{2} C_{5}\left[1+\left\|\mathbf{u}_{h}\right\|_{W^{1, r}}+\left\|\mathbf{v}_{h}\right\|_{W^{1, r}}\right]^{2-r}\left\|p-q_{h}\right\|_{L^{r^{\prime}}(\Omega)}^{2}
\end{aligned}
$$

By moving (1/2) $\left\|\left|\mathbf{u}-\mathbf{u}_{h} \|\right|^{2}\right.$ to the left-hand side, we obtain (4.11). From the inf-sup condition (4.1), we have, for all $q_{h} \in Q_{h}$,

$$
C_{h}\left\|q_{h}-p_{h}\right\|_{L^{\kappa^{\prime}}}<\sup _{\mathbf{v}_{h} \in V_{h}} \frac{\int_{\Omega}\left(q_{h}-p_{h}\right) \operatorname{div}\left(\mathbf{v}_{h}\right) d V}{\left\|\mathbf{v}_{h}\right\|_{W^{1, \kappa}}}
$$

From (4.10), we have, for all $\mathbf{v}_{h} \in V_{h}$,

$$
\begin{aligned}
& \int_{\Omega} \operatorname{div}\left(\mathbf{v}_{h}\right)\left(q_{h}-p_{h}\right) d V \\
& \quad=\int_{\Omega} 2\left(\mu(|\varepsilon(\mathbf{u})|) \varepsilon(\mathbf{u})-\mu\left(\left|\varepsilon\left(\mathbf{u}_{h}\right)\right|\right) \varepsilon\left(\mathbf{u}_{h}\right)\right): \varepsilon\left(\mathbf{v}_{h}\right) d V+\int_{\Omega} \operatorname{div}\left(\mathbf{v}_{h}\right)\left(q_{h}-p\right) d V .
\end{aligned}
$$


From (4.19), (4.20), and (3.5) with $1-1 / n=2-r$, there exist $C_{6}, C_{7}>0$ such that

$$
\begin{aligned}
& C_{h}\left\|q_{h}-p_{h}\right\|_{L^{\kappa^{\prime}}} \\
& \quad<\sup _{\mathbf{v}_{h} \in V_{h}} \frac{\left|\int_{\Omega} 2\left(\mu(|\varepsilon(\mathbf{u})|) \varepsilon(\mathbf{u})-\mu\left(\left|\varepsilon\left(\mathbf{u}_{h}\right)\right|\right) \varepsilon\left(\mathbf{u}_{h}\right)\right): \varepsilon\left(\mathbf{v}_{h}\right) d V\right|}{\left\|\mathbf{v}_{h}\right\|_{W^{1, \kappa}}}+\sup _{\mathbf{v}_{h} \in V_{h}} \frac{\left|\int_{\Omega} \operatorname{div}\left(\mathbf{v}_{h}\right)\left(q_{h}-p\right) d V\right|}{\left\|\mathbf{v}_{h}\right\|_{W^{1, \kappa}}} \\
& \quad \leq C_{6}\left\{\left\|2\left(\mu(|\varepsilon(\mathbf{u})|) \varepsilon(\mathbf{u})-\mu\left(\left|\varepsilon\left(\mathbf{u}_{h}\right)\right|\right) \varepsilon\left(\mathbf{u}_{h}\right)\right)\right\|_{L^{\kappa^{\prime}}}+\left\|q_{h}-p\right\|_{L^{\kappa^{\prime}}}\right\} \\
& \quad \leq C_{7}\left\{\int_{\Omega}\left(1+|\varepsilon(\mathbf{u})|+\left|\varepsilon\left(\mathbf{u}-\mathbf{u}_{h}\right)\right|\right)^{(r-2) \kappa^{\prime}}\left|\varepsilon\left(\mathbf{u}-\mathbf{u}_{h}\right)\right|^{\kappa^{\prime}} d V\right\}^{1 / \kappa^{\prime}}+C_{6}\left\|q_{h}-p\right\|_{L^{\kappa^{\prime}}} \\
& \quad \leq C_{7} C_{8}\left\{\int_{\Omega}\left(1+|\varepsilon(\mathbf{u})|+\left|\varepsilon\left(\mathbf{u}-\mathbf{u}_{h}\right)\right|\right)^{(r-2)}\left|\varepsilon\left(\mathbf{u}-\mathbf{u}_{h}\right)\right|^{2} d V\right\}^{1 / \kappa^{\prime}}+C_{6}\left\|q_{h}-p\right\|_{L^{\kappa^{\prime}}} \\
& \quad \leq C_{7}\left\|\left|\mathbf{u}-\mathbf{u}_{h}\right|\right\|^{2 / \mathcal{\kappa}^{\prime}}+C_{6}\left\|q_{h}-p\right\|_{L^{\kappa^{\prime}}},
\end{aligned}
$$

where $C_{8}:=\left\|\left(1+|\varepsilon(\mathbf{u})|+\left|\varepsilon\left(\mathbf{u}-\mathbf{u}_{h}\right)\right|\right)^{(r-2)}\left|\varepsilon\left(\mathbf{u}-\mathbf{u}_{h}\right)\right|^{2-\kappa}\right\|_{L^{\infty}}^{1 / \kappa}<1$. Eventually, the previous inequality together with $\left\|p-p_{h}\right\|_{L^{x^{\prime}}} \leq\left\|q_{h}-p_{h}\right\|_{L^{x^{\prime}}}+\left\|p-q_{h}\right\|_{L^{x^{\prime}}}$ leads to (4.12).

Theorem 4.8. Let $(\mathbf{u}, p) \in(V, Q)$ be the solution of (3.8), and $\mathbf{u}_{h} \in V_{\mathrm{div}, h}$ the solution of (4.4). For all $\left(\mathbf{v}_{h}, q_{h}\right) \in V_{\mathrm{div}, h} \times Q_{h}$ and for all $\kappa \in[r, 2]$, assuming $\mathbf{u} \in W^{1, \kappa}(\Omega)$, one has

$$
\left\|\mathbf{u}-\mathbf{u}_{h}\right\|_{W^{1, r}} \leq D_{1}\left[1+\left\|\mathbf{u}_{h}\right\|_{W^{1, r}}+\left\|\mathbf{v}_{h}\right\|_{W^{1, r}}\right]^{2-r}\left(\left\|\mathbf{u}-\mathbf{v}_{h}\right\|_{W^{1, \kappa}}^{\kappa / 2}+\left\|p-q_{h}\right\|_{L^{r^{\prime}}}\right),
$$

where $D_{1}>0$. Moreover, if the spaces $V_{h}$ and $Q_{h}$ satisfy the inf-sup condition (4.1), then the solution $\left(\mathbf{u}_{h}, p_{h}\right)$ of (4.2) satisfies for all $\left(\mathbf{v}_{h}, q_{h}\right) \in V_{h} \times Q_{h}$ and for all $\kappa \in[r, 2]$, assuming $\mathbf{u} \in W^{1, \kappa}(\Omega)$,

$$
\begin{aligned}
\left\|\mathbf{u}-\mathbf{u}_{h}\right\|_{W^{1, r}} \leq D_{2}[1 & \left.+\left\|\mathbf{u}_{h}\right\|_{W^{1, r}}+\left\|\mathbf{v}_{h}\right\|_{W^{1, r}}\right]^{2-r}\left(\left\|\mathbf{u}-\mathbf{v}_{h}\right\|_{W^{1, \kappa}}^{\kappa / 2}+\left\|p-q_{h}\right\|_{L^{r^{\prime}}}\right), \\
\left\|p-p_{h}\right\|_{L^{\kappa^{\prime}}} \leq D_{3}[1 & \left.+\left\|\mathbf{u}_{h}\right\|_{W^{1, r}}+\left\|\mathbf{v}_{h}\right\|_{W^{1, r}}\right]^{(2-r) / \mathcal{\kappa}^{\prime}} \\
& \times\left\{\left(\left\|\mathbf{u}-\mathbf{v}_{h}\right\|_{W^{1, \kappa}}^{\kappa / 2}+\left\|p-q_{h}\right\|_{L^{r^{\prime}}}\right)^{2 / \mathcal{K}^{\prime}}+\left\|p-q_{h}\right\|_{L^{\kappa^{\prime}}}\right\},
\end{aligned}
$$

where $D_{2}, D_{3}>0$. The constants $D_{1}, D_{2}, D_{3}$ do not depend on $\mathbf{u}_{h}$ and $\mathbf{v}_{h}$; however, $D_{2}$ and $D_{3}$ increasingly depends on $\left(C_{h}\right)^{-1}$.

Proof. On one hand, inequality (4.22) follows from inequalities (4.7), (4.11), and (4.8). On the other hand, (4.23) follows from (4.22) and from the following property (see (1.16), page 115 in [16]): for all $\mathbf{v} \in V_{\text {div }}$ and for all $\mathbf{w}_{h} \in V_{h}$, there exists $\mathbf{v}_{h} \in V_{\text {div }, h}$ such that

$$
\left\|\mathbf{v}-\mathbf{v}_{h}\right\|_{W^{1, \kappa}} \leq C\left\|\mathbf{v}-\mathbf{w}_{h}\right\|_{W^{1, \kappa}}
$$

where $C$ depends on the inf-sup constant $C_{h}$. Eventually, (4.24) follows from (4.12), (4.11), and (4.8). 
Theorem 4.9. Assume that, for all $\kappa \in[r, 2]$, there exists a continuous operator $\pi_{h}:\left[W^{2, \kappa}\right]^{d} \rightarrow V_{h}$ that satisfies

$$
\left\|\mathbf{u}-\pi_{h}(\mathbf{u})\right\|_{W^{1, \kappa}} \leq C h\|\mathbf{u}\|_{W^{2, \kappa},}, \forall \mathbf{u} \in\left[W^{2, \kappa}\right]^{d},
$$

and a continuous operator $\rho_{h}: W^{1, \kappa^{\prime}} \rightarrow Q_{h}$ that satisfies

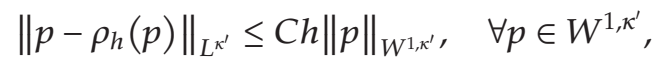

where $h$ is the size of the higher diameter of the elements of $\tau_{h}$. Assume that $V_{h}$ and $Q_{h}$ satisfy the inf-sup condition (4.1). Let $(\mathbf{u}, p)$ be the solution of problem (3.8) and let $\left(\mathbf{u}_{h}, p_{h}\right)$ be the solution of problem (4.2). Assume that $(\mathbf{u}, p) \in\left(\left[W^{2, \kappa}\right]^{d}, W^{1, \kappa^{\prime}}\right)$, where $\kappa \in[r, 2]$, then one has

$$
\left\|\mathbf{u}-\mathbf{u}_{h}\right\|_{W^{1, r}}+\left(\left\|p-p_{h}\right\|_{L^{\kappa^{\prime}}}\right)^{\kappa^{\prime} / 2} \leq D h^{\kappa / 2}
$$

where $D=D\left(\|\mathbf{u}\|_{W^{2, \kappa},},\|p\|_{W^{1, r^{\prime}},}\left(C_{h}\right)^{-1}\right)>0$.

Proof. Apply (4.23) and (4.24) with $\mathbf{v}_{h}=\pi_{h}(\mathbf{u})$ and $q_{h}=\rho_{h}(p)$. By using the continuity of $\pi_{h}$, (4.5), (4.26), and (4.27), there exist $C_{1}, C_{2}, C_{3}, C_{4}>0$ such that

$$
\begin{aligned}
& \left\|\mathbf{u}-\mathbf{u}_{h}\right\|_{W^{1, r}} \leq C_{1}\left[1+\|\mathbf{f}\|_{L^{r^{\prime}}}+\|\mathbf{u}\|_{W^{1, r}}\right]^{2-r}\left(h^{(\kappa / 2)}+h\right) \leq C_{2} h^{(\kappa / 2)}, \\
& \left\|p-p_{h}\right\|_{L^{\kappa^{\prime}}} \leq C_{3}\left[1+\|\mathbf{f}\|_{L^{r^{\prime}}}+\|\mathbf{u}\|_{W^{1, r},}\right]^{(2-r) / \kappa^{\prime}}\left\{\left(h^{(\kappa / 2)}+h\right)^{\left(2 / \kappa^{\prime}\right)}+h\right\} \leq C_{4} h^{\left(\kappa / \kappa^{\prime}\right)} .
\end{aligned}
$$

The estimate (4.28) directly follows from (4.29).

Remark 4.10. The combination $\left[\mathbb{P}_{1} / \text { Bulle }\right]^{d}-\mathbb{P}_{1}$ for spaces $V_{h}$ and $Q_{h}$, introduced in [23], satisfies the assumptions of Theorem 4.9; see Lemma 4.20 page 190 of [15] for the inf-sup condition (4.1) and $[15,16]$ for the interpolation inequalities (4.26) and (4.27).

Remark 4.11. If $\Gamma_{R} \neq \emptyset$, a similar analysis can be led by replacing the norm defined by (4.6) by

$$
\mathbf{v} \longrightarrow\|\mathbf{v} \mid\|:=\sqrt{\int_{\Omega} \frac{|\varepsilon(\mathbf{v})|^{2}}{(1+|\varepsilon(\mathbf{u})|+|\varepsilon(\mathbf{v})|)^{2-r}} d V}+\sqrt{\int_{\Gamma_{R}} \frac{|\mathbf{v}|^{2}}{(1+|\mathbf{u}|+|\mathbf{v}|)^{2-r}} d S} .
$$

\section{Successive Approximations}

In this section, several successive approximation algorithms are proposed for solving the nonlinearity of the discrete problem (4.4) when $n>1$. For the sake of simplicity, we suppose $\Gamma_{R}=\emptyset$ in this section; see Remark 5.8. We present a unified scheme that contains the classical fixed point method together with Newton's method. The mesh $\tau_{h}$ is fixed, and we assume that the approximation spaces satisfy $V_{h} \subset V \cap\left[W^{1, \infty}(\Omega)\right]^{d}$ and $Q_{h} \subset Q \cap L^{\infty}(\Omega)$. In what 
follows, $\|\cdot\|$ denotes an arbitrary norm of $V_{\text {div }, h}$. Since $V_{\text {div }, h}$ is a finite-dimensional space, all norms are equivalent. Let $\gamma \in[0,1]$. We define

$$
E: \begin{cases}V_{\mathrm{div}, h} & \longrightarrow V_{\mathrm{div}, h}, \\ \tilde{\mathbf{u}}_{h} & \longrightarrow \tilde{\mathbf{w}}_{h},\end{cases}
$$

where $\tilde{\mathbf{w}}_{h} \in V_{\mathrm{div}, h}$ solves

$$
\begin{aligned}
& 2 \int_{\Omega} \mu\left(\left|\varepsilon\left(\tilde{\mathbf{u}}_{h}\right)\right|\right) \varepsilon\left(\widetilde{\mathbf{w}}_{h}\right): \varepsilon\left(\mathbf{v}_{h}\right) d V+2 \gamma \int_{\Omega} \frac{\mu^{\prime}\left(\left|\varepsilon\left(\tilde{\mathbf{u}}_{h}\right)\right|\right)}{\left|\varepsilon\left(\widetilde{\mathbf{u}}_{h}\right)\right|}\left(\varepsilon\left(\tilde{\mathbf{u}}_{h}\right): \varepsilon\left(\tilde{\mathbf{w}}_{h}-\widetilde{\mathbf{u}}_{h}\right)\right)\left(\varepsilon\left(\tilde{\mathbf{u}}_{h}\right): \varepsilon\left(\mathbf{v}_{h}\right)\right) d V \\
& \quad=\int_{\Omega} \mathbf{g} \cdot \mathbf{v}_{h} d V, \quad \forall \mathbf{v}_{h} \in V_{\mathrm{div}, h} .
\end{aligned}
$$

The application $E$ is well defined. Indeed, by using, respectively, $\mu^{\prime}<0$, inequalities (3.3) and (3.2), there exist $C_{1}, C_{2}>0$ such that

$$
\begin{gathered}
2 \int_{\Omega} \mu\left(\left|\varepsilon\left(\widetilde{\mathbf{u}}_{h}\right)\right|\right)\left|\varepsilon\left(\widetilde{\mathbf{w}}_{h}\right)\right|^{2} d V+2 \gamma \int_{\Omega} \frac{\mu^{\prime}\left(\left|\varepsilon\left(\widetilde{\mathbf{u}}_{h}\right)\right|\right)}{\left|\varepsilon\left(\widetilde{\mathbf{u}}_{h}\right)\right|}\left(\varepsilon\left(\widetilde{\mathbf{u}}_{h}\right): \varepsilon\left(\widetilde{\mathbf{w}}_{h}\right)\right)^{2} d V \\
\quad \geq 2 \int_{\Omega} \mu\left(\left|\varepsilon\left(\widetilde{\mathbf{u}}_{h}\right)\right|\right)\left|\varepsilon\left(\widetilde{\mathbf{w}}_{h}\right)\right|^{2} d V-2 \gamma(1-1 / n) \int_{\Omega} \mu\left(\left|\varepsilon\left(\widetilde{\mathbf{u}}_{h}\right)\right|\right)\left|\varepsilon\left(\widetilde{\mathbf{w}}_{h}\right)\right|^{2} d V \\
\geq \frac{2 C_{1}(1-\gamma(1-1 / n))}{\left(1+\left\|\varepsilon\left(\widetilde{\mathbf{u}}_{h}\right)\right\|_{L^{\infty}}\right)^{1-1 / n}} \int_{\Omega}\left|\varepsilon\left(\widetilde{\mathbf{w}}_{h}\right)\right|^{2} d V \geq C_{2}\left\|\widetilde{\mathbf{w}}_{h}\right\|^{2} .
\end{gathered}
$$

As a consequence, the problem (5.2) is coercive. From the Lax-Milgram Theorem, see [15] page 83 , there exists a unique solution $\widetilde{\mathbf{w}}_{h} \in V_{\mathrm{div}, h}$ of (5.2).

In what follows, $\mathbf{u}_{h}$ denotes the solution (4.4), which is also the unique fixed point of $E$. Assume that $\mathbf{u}_{h, 0}$ is given; we define iteratively a sequence $\mathbf{u}_{h, k}$, for all $k \geq 1$, by

$$
\mathbf{u}_{h, k+1}=E\left(\mathbf{u}_{h, k}\right)
$$

Our goal is to prove that $\mathbf{u}_{h, k}$ converges to $\mathbf{u}_{h}$ when $k$ goes to the infinity. When $\gamma=0$, we obtain the classical fixed point method, widely used to solve the nonlinearity of Glen's law; see $[6,9,14]$. When $\gamma=1$, we have an additional term in (5.2) which corresponds to Newton's method; see Remark 5.5. The case $\gamma \in(0,1)$ corresponds to a hybrid fixed pointNewton's method. The convergence of sequence $\mathbf{u}_{h, k}$ requires several preliminary results. We compute the first derivative of $E$ in Lemma 5.1. Lemma 5.2 provides an upper bound of the first derivative. Eventually, Theorem 5.3 states the linear convergence of $\mathbf{u}_{h, k}$ by using the Banach fixed point theorem. Theorem 5.7 states the second-order convergence when $\gamma=1$. By differentiating formally (5.2) at point $\widetilde{\mathbf{u}}_{h}$ in direction $\overline{\mathbf{u}}_{h}$, with $\widetilde{\mathbf{w}}_{h}=E\left(\widetilde{\mathbf{u}}_{h}\right)$, we obtain the following lemma. 
Lemma 5.1. Let $\left(\widetilde{\mathbf{u}}_{h}, \widetilde{\mathbf{w}}_{h}\right)$ satisfy $E\left(\widetilde{\mathbf{u}}_{h}\right)=\widetilde{\mathbf{w}}_{h}$. The application $E$ is Gateaux differentiable at point $\widetilde{\mathbf{u}}_{h}$ and its derivative is given by

$$
D E\left(\tilde{\mathbf{u}}_{h}\right): \begin{cases}V_{\mathrm{div}, h} & \longrightarrow V_{\mathrm{div}, h}, \\ \overline{\mathbf{u}}_{h} & \longrightarrow \overline{\mathbf{w}}_{h},\end{cases}
$$

where $\overline{\mathbf{w}}_{h}$ solves

$$
\begin{aligned}
& 2 \int_{\Omega} \mu\left(\left|\varepsilon\left(\tilde{\mathbf{u}}_{h}\right)\right|\right) \varepsilon\left(\overline{\mathbf{w}}_{h}\right): \varepsilon\left(\mathbf{v}_{h}\right) d V+2 \int_{\Omega} \frac{\mu^{\prime}\left(\left|\varepsilon\left(\tilde{\mathbf{u}}_{h}\right)\right|\right)}{\left|\varepsilon\left(\widetilde{\mathbf{u}}_{h}\right)\right|}\left(\varepsilon\left(\tilde{\mathbf{u}}_{h}\right): \varepsilon\left(\overline{\mathbf{u}}_{h}\right)\right)\left(\varepsilon\left(\tilde{\mathbf{w}}_{h}\right): \varepsilon\left(\mathbf{v}_{h}\right)\right) d V \\
& +\gamma\left[2 \int_{\Omega} \frac{\mu^{\prime \prime}\left(\left|\varepsilon\left(\widetilde{\mathbf{u}}_{h}\right)\right|\right)\left|\varepsilon\left(\widetilde{\mathbf{u}}_{h}\right)\right|-\mu^{\prime}\left(\left|\varepsilon\left(\widetilde{\mathbf{u}}_{h}\right)\right|\right)}{\left|\varepsilon\left(\tilde{\mathbf{u}}_{h}\right)\right|^{3}}\left(\varepsilon\left(\widetilde{\mathbf{u}}_{h}\right): \varepsilon\left(\overline{\mathbf{u}}_{h}\right)\right)\right. \\
& \left.\times\left(\varepsilon\left(\tilde{\mathbf{u}}_{h}\right): \varepsilon\left(\tilde{\mathbf{w}}_{h}-\tilde{\mathbf{u}}_{h}\right)\right)\left(\varepsilon\left(\tilde{\mathbf{u}}_{h}\right): \varepsilon\left(\mathbf{v}_{h}\right)\right) d V\right] \\
& +\gamma\left[2 \int_{\Omega} \frac{\mu^{\prime}\left(\left|\varepsilon\left(\widetilde{\mathbf{u}}_{h}\right)\right|\right)}{\left|\varepsilon\left(\widetilde{\mathbf{u}}_{h}\right)\right|}\left(\varepsilon\left(\overline{\mathbf{u}}_{h}\right): \varepsilon\left(\widetilde{\mathbf{w}}_{h}-\widetilde{\mathbf{u}}_{h}\right)\right)\left(\varepsilon\left(\tilde{\mathbf{u}}_{h}\right): \varepsilon\left(\mathbf{v}_{h}\right)\right) d V\right] \\
& +\gamma\left[2 \int_{\Omega} \frac{\mu^{\prime}\left(\left|\varepsilon\left(\widetilde{\mathbf{u}}_{h}\right)\right|\right)}{\left|\varepsilon\left(\widetilde{\mathbf{u}}_{h}\right)\right|}\left(\varepsilon\left(\widetilde{\mathbf{u}}_{h}\right): \varepsilon\left(\overline{\mathbf{w}}_{h}-\overline{\mathbf{u}}_{h}\right)\right)\left(\varepsilon\left(\widetilde{\mathbf{u}}_{h}\right): \varepsilon\left(\mathbf{v}_{h}\right)\right) d V\right] \\
& +\gamma\left[2 \int_{\Omega} \frac{\mu^{\prime}\left(\left|\varepsilon\left(\widetilde{\mathbf{u}}_{h}\right)\right|\right)}{\left|\varepsilon\left(\widetilde{\mathbf{u}}_{h}\right)\right|}\left(\varepsilon\left(\widetilde{\mathbf{u}}_{h}\right): \varepsilon\left(\widetilde{\mathbf{w}}_{h}-\widetilde{\mathbf{u}}_{h}\right)\right)\left(\varepsilon\left(\overline{\mathbf{u}}_{h}\right): \varepsilon\left(\mathbf{v}_{h}\right)\right) d V\right]=0, \quad \forall \mathbf{v}_{h} \in V_{\mathrm{div}, h} .
\end{aligned}
$$

The problems (5.2) and (5.6) have the same coercivity properties to compute $\overline{\mathbf{w}}_{h}$ (resp., $\widetilde{\mathbf{w}}_{h}$ ) from $\overline{\mathbf{u}}_{h}$ (resp., $\tilde{\mathbf{u}}_{h}$ ). As a consequence, the problem (5.6) is well-posed by the LaxMilgram theorem. To prove the convergence of the sequence $\mathbf{u}_{h, k}$, we look for a norm that makes $E$ a contraction at point $\mathbf{u}_{h}$.

Lemma 5.2. Let $\gamma \in[0,1]$, and let $\mathbf{u}_{h}$ be the fixed point of $E$. The application $D E\left(\mathbf{u}_{h}\right)$ satisfies

$$
\left\|\left|D E\left(\mathbf{u}_{h}\right)\right|\right\|_{\mu} \leq \frac{(1-\gamma)(1-1 / n)}{1-(1-1 / n) \gamma}<1,
$$

where $\||\cdot|\|_{\mu}$ is the subordinated norm to $\|\cdot\|_{\mu}:=\sqrt{\int_{\Omega} \mu\left(\left|\varepsilon\left(\mathbf{u}_{h}\right)\right|\right)|\varepsilon(\cdot)|^{2} d V}$.

Proof. Since $E\left(\mathbf{u}_{h}\right)=\mathbf{u}_{h}$, then (5.6), with $\widetilde{\mathbf{u}}_{h}=\mathbf{u}_{h}$ and $\widetilde{\mathbf{w}}_{\mathbf{h}}=\mathbf{u}_{h}$, is rewriten as

$$
\begin{aligned}
& \int_{\Omega} \mu\left(\left|\varepsilon\left(\mathbf{u}_{h}\right)\right|\right) \varepsilon\left(\overline{\mathbf{w}}_{h}\right): \varepsilon\left(\mathbf{v}_{h}\right) d V \\
& \quad+(1-\gamma)\left[\int_{\Omega} \frac{\mu^{\prime}\left(\left|\varepsilon\left(\mathbf{u}_{h}\right)\right|\right)}{\left|\varepsilon\left(\mathbf{u}_{h}\right)\right|}\left(\varepsilon\left(\mathbf{u}_{h}\right): \varepsilon\left(\overline{\mathbf{u}}_{h}\right)\right)\left(\varepsilon\left(\mathbf{u}_{h}\right): \varepsilon\left(\mathbf{v}_{h}\right)\right) d V\right] \\
& \quad+\gamma\left[\int_{\Omega} \frac{\mu^{\prime}\left(\left|\varepsilon\left(\mathbf{u}_{h}\right)\right|\right)}{\left|\varepsilon\left(\mathbf{u}_{h}\right)\right|}\left(\varepsilon\left(\mathbf{u}_{h}\right): \varepsilon\left(\overline{\mathbf{w}}_{h}\right)\right)\left(\varepsilon\left(\mathbf{u}_{h}\right): \varepsilon\left(\mathbf{v}_{h}\right)\right) d V\right]=0,
\end{aligned}
$$


for all $\mathbf{v}_{h} \in V_{\mathrm{div}, h}$. From (5.8), $\mu^{\prime}<0$, and (3.3), we have

$$
\begin{aligned}
\int_{\Omega} \mu\left(\left|\varepsilon\left(\mathbf{u}_{h}\right)\right|\right) \varepsilon\left(\overline{\mathbf{w}}_{h}\right): \varepsilon\left(\mathbf{v}_{h}\right) d V \leq & -(1-\gamma)\left[\int_{\Omega} \mu^{\prime}\left(\left|\varepsilon\left(\mathbf{u}_{h}\right)\right|\right)\left|\varepsilon\left(\mathbf{u}_{h}\right)\right|\left|\varepsilon\left(\overline{\mathbf{u}}_{h}\right)\right|\left|\varepsilon\left(\mathbf{v}_{h}\right)\right| d V\right] \\
& -\gamma\left[\int_{\Omega} \mu^{\prime}\left(\left|\varepsilon\left(\mathbf{u}_{h}\right)\right|\right)\left|\varepsilon\left(\mathbf{u}_{h}\right)\right|\left|\varepsilon\left(\overline{\mathbf{w}}_{h}\right) \| \varepsilon\left(\mathbf{v}_{h}\right)\right| d V\right] \\
\leq & (1-\gamma)\left(1-\frac{1}{n}\right)\left[\int_{\Omega} \mu\left(\left|\varepsilon\left(\mathbf{u}_{h}\right)\right|\right)\left|\varepsilon\left(\overline{\mathbf{u}}_{h}\right)\right|\left|\varepsilon\left(\mathbf{v}_{h}\right)\right| d V\right] \\
& +\gamma\left(1-\frac{1}{n}\right)\left[\int_{\Omega} \mu\left(\left|\varepsilon\left(\mathbf{u}_{h}\right)\right|\right)\left|\varepsilon\left(\overline{\mathbf{w}}_{h}\right) \| \varepsilon\left(\mathbf{v}_{h}\right)\right| d V\right]
\end{aligned}
$$

By setting $\mathbf{v}_{h}=\overline{\mathbf{w}}_{h}$ in (5.9), we obtain

$$
\left\|\overline{\mathbf{w}}_{h}\right\|_{\mu}^{2} \leq(1-\gamma)\left(1-\frac{1}{n}\right) \int_{\Omega} \mu\left(\left|\varepsilon\left(\mathbf{u}_{h}\right)\right|\right)\left|\varepsilon\left(\overline{\mathbf{u}}_{h}\right)\left\|\varepsilon\left(\overline{\mathbf{w}}_{h}\right) \mid d V+\gamma\left(1-\frac{1}{n}\right)\right\| \overline{\mathbf{w}}_{h} \|_{\mu}^{2}\right.
$$

From (5.10) and Cauchy-Schwarz's inequality, we obtain

$$
\left\|\overline{\mathbf{w}}_{h}\right\|_{\mu}^{2} \leq\left[\frac{(1-\gamma)(1-1 / n)}{1-(1-1 / n) \gamma}\right]\left\|\overline{\mathbf{u}}_{h}\right\|_{\mu}\left\|\overline{\mathbf{w}}_{h}\right\|_{\mu} .
$$

Eventually, (5.7) follows from the definition of norm ||$|\cdot| \|_{\mu}$.

Theorem 5.3. Let $\gamma \in[0,1]$, let $\tau_{h}$ be a given mesh of $\Omega$, and let $\|\cdot\|$ be a norm of $V_{\operatorname{div}, h}$. There exist $\delta>0$ and $C>0$ such that if $\left\|\mathbf{u}_{h, 0}-\mathbf{u}_{h}\right\|<\delta$, then one has

$$
\left\|\mathbf{u}_{h, k}-\mathbf{u}_{h}\right\| \leq C\left[\frac{(1-\gamma)(1-1 / n)}{1-(1-1 / n) \gamma}\right]^{k}\left\|\mathbf{u}_{h, 0}-\mathbf{u}_{h}\right\|,
$$

for all $k \geq 0$, and $\mathbf{u}_{h, k}$ is linearly convergent to $\mathbf{u}_{h}$.

Proof. From Lemma 5.2, the spectral radius of $D E\left(\mathbf{u}_{h}\right)$ is lower than constant:

$$
\left[\frac{(1-\gamma)(1-1 / n)}{1-(1-1 / n) \gamma}\right]
$$

which is lower than 1. The theorem is then a direct application of the Banach fixed point theorem in $V_{\text {div, } h \text {. }}$

Remark 5.4. In Theorem 5.3, it should be stressed that $\delta$ depends on $h$. When $h \rightarrow 0$ (i.e., if we replace $\mathbf{u}_{h}$ by $\mathbf{u}$ ), we cannot ensure Theorem 5.3 to remain true. Nevertheless, in practise, $\delta$ seems to be independent of $h$, see Section 6 . 
When $\gamma=1$, we have $D E\left(\mathbf{u}_{h}\right)=\mathbf{0}$ from Lemma 5.2. It suggests that the convergence of sequence $\mathbf{u}_{h, k}$ is quadratic. To establish the second-order convergence, we define, for all $\mathbf{v}_{h} \in V_{\operatorname{div}, h}$, the application $\mathcal{F}\left(\cdot, \mathbf{v}_{h}\right): V_{\mathrm{div}, h} \rightarrow \mathbb{R}$ by

$$
\mathcal{F}\left(\widetilde{\mathbf{u}}_{h} ; \mathbf{v}_{h}\right):=2 \int_{\Omega} \mu\left(\left|\varepsilon\left(\widetilde{\mathbf{u}}_{h}\right)\right|\right) \varepsilon\left(\widetilde{\mathbf{u}}_{h}\right): \varepsilon\left(\mathbf{v}_{h}\right) d V-\int_{\Omega} \mathbf{f} \cdot \mathbf{v}_{h} d V .
$$

Let $\widetilde{\mathbf{u}}_{h}, \overline{\mathbf{u}}_{h}, \overline{\overline{\mathbf{u}}}_{h} \in V_{\mathrm{div}, h}$. We compute formally the first-order derivative of $\mathcal{F}$ at point $\widetilde{\mathbf{u}}_{h}$ in direction $\overline{\mathbf{u}}_{h}$ :

$$
\begin{aligned}
\left\langle D \mathcal{F}\left(\tilde{\mathbf{u}}_{h} ; \mathbf{v}_{h}\right), \overline{\mathbf{u}}_{h}\right\rangle= & 2 \int_{\Omega} \mu\left(\left|\varepsilon\left(\tilde{\mathbf{u}}_{h}\right)\right|\right) \varepsilon\left(\overline{\mathbf{u}}_{h}\right): \varepsilon\left(\mathbf{v}_{h}\right) d V \\
& +2 \int_{\Omega} \frac{\mu^{\prime}\left(\left|\varepsilon\left(\tilde{\mathbf{u}}_{h}\right)\right|\right)}{\left|\varepsilon\left(\widetilde{\mathbf{u}}_{h}\right)\right|}\left(\varepsilon\left(\widetilde{\mathbf{u}}_{h}\right): \varepsilon\left(\overline{\mathbf{u}}_{h}\right)\right)\left(\varepsilon\left(\widetilde{\mathbf{u}}_{h}\right): \varepsilon\left(\mathbf{v}_{h}\right)\right) d V,
\end{aligned}
$$

and the second-order derivative of $\mathcal{F}$ at point $\tilde{\mathbf{u}}_{h}$ in direction $\left(\overline{\mathbf{u}}_{h}, \overline{\mathbf{u}}_{h}\right)$ :

$$
\begin{aligned}
& \left\langle D^{2} \boldsymbol{F}\left(\widetilde{\mathbf{u}}_{h} ; \mathbf{v}_{h}\right), \overline{\mathbf{u}}_{h}, \overline{\overline{\mathbf{u}}}_{h}\right\rangle=2 \int_{\Omega} \frac{\mu^{\prime}\left(\left|\varepsilon\left(\widetilde{\mathbf{u}}_{h}\right)\right|\right)}{\left|\varepsilon\left(\widetilde{\mathbf{u}}_{h}\right)\right|}\left(\varepsilon\left(\widetilde{\mathbf{u}}_{h}\right): \varepsilon\left(\overline{\overline{\mathbf{u}}}_{h}\right)\right)\left(\varepsilon\left(\overline{\mathbf{u}}_{h}\right): \varepsilon\left(\mathbf{v}_{h}\right)\right) d V \\
& +2 \int_{\Omega} \frac{\mu^{\prime \prime}\left(\left|\varepsilon\left(\tilde{\mathbf{u}}_{h}\right)\right|\right)\left|\varepsilon\left(\tilde{\mathbf{u}}_{h}\right)\right|-\mu^{\prime}\left(\left|\varepsilon\left(\tilde{\mathbf{u}}_{h}\right)\right|\right)}{\left|\varepsilon\left(\tilde{\mathbf{u}}_{h}\right)\right|^{3}} \\
& \times\left(\varepsilon\left(\tilde{\mathbf{u}}_{h}\right): \varepsilon\left(\overline{\overline{\mathbf{u}}}_{h}\right)\right)\left(\varepsilon\left(\tilde{\mathbf{u}}_{h}\right): \varepsilon\left(\overline{\mathbf{u}}_{h}\right)\right)\left(\varepsilon\left(\tilde{\mathbf{u}}_{h}\right): \varepsilon\left(\mathbf{v}_{h}\right)\right) d V \\
& +2 \int_{\Omega} \frac{\mu^{\prime}\left(\left|\varepsilon\left(\widetilde{\mathbf{u}}_{h}\right)\right|\right)}{\left|\varepsilon\left(\widetilde{\mathbf{u}}_{h}\right)\right|}\left(\varepsilon\left(\overline{\overline{\mathbf{u}}}_{h}\right): \varepsilon\left(\overline{\mathbf{u}}_{h}\right)\right)\left(\varepsilon\left(\widetilde{\mathbf{u}}_{h}\right): \varepsilon\left(\mathbf{v}_{h}\right)\right) d V \\
& +2 \int_{\Omega} \frac{\mu^{\prime}\left(\left|\varepsilon\left(\widetilde{\mathbf{u}}_{h}\right)\right|\right)}{\left|\varepsilon\left(\widetilde{\mathbf{u}}_{h}\right)\right|}\left(\varepsilon\left(\widetilde{\mathbf{u}}_{h}\right): \varepsilon\left(\overline{\mathbf{u}}_{h}\right)\right)\left(\varepsilon\left(\overline{\overline{\mathbf{u}}}_{h}\right): \varepsilon\left(\mathbf{v}_{h}\right)\right) d V .
\end{aligned}
$$

Remark 5.5. If $\gamma=1$, we have, from the definition of $E$ (5.2), of $\mathcal{F}(5.14)$, and of $D \mathcal{F}$ (5.15),

$$
\mathbf{u}_{h, k+1}=E\left(\mathbf{u}_{h, k}\right) \Longleftrightarrow \mathcal{F}\left(\mathbf{u}_{h, k} ; \mathbf{v}_{h}\right)+\left\langle D \mathcal{F}\left(\mathbf{u}_{h, k} ; \mathbf{v}_{h}\right), \mathbf{u}_{h, k+1}-\mathbf{u}_{h, k}\right\rangle=0, \quad \forall \mathbf{v}_{h} \in V_{\mathrm{div}, h},
$$

which highlights Newton's method.

Lemma 5.6. The following inequalities hold, for all $\widetilde{\mathbf{u}}_{h}, \overline{\mathbf{u}}_{h}, \overline{\overline{\mathbf{u}}}_{h} \in V_{\mathrm{div}, h}$ :

$$
\begin{gathered}
\frac{2}{n} \int_{\Omega} \mu\left(\left|\varepsilon\left(\tilde{\mathbf{u}}_{h}\right)\right|\right)\left|\varepsilon\left(\overline{\mathbf{u}}_{h}\right)\right|^{2} d V \leq\left\langle D \mathcal{F}\left(\widetilde{\mathbf{u}}_{h} ; \overline{\mathbf{u}}_{h}\right), \overline{\mathbf{u}}_{h}\right\rangle \\
-\left\langle D^{2} \mathcal{F}\left(\widetilde{\mathbf{u}}_{h} ; \overline{\mathbf{u}}_{h}\right), \overline{\overline{\mathbf{u}}}_{h}, \overline{\overline{\mathbf{u}}}_{h}\right\rangle \leq \int_{\Omega}\left(8\left|\mu^{\prime}\left(\left|\varepsilon\left(\widetilde{\mathbf{u}}_{h}\right)\right|\right)\right|+2\left|\mu^{\prime \prime}\left(\left|\varepsilon\left(\widetilde{\mathbf{u}}_{h}\right)\right|\right)\right|\left|\varepsilon\left(\widetilde{\mathbf{u}}_{h}\right)\right|\right)\left|\varepsilon\left(\overline{\overline{\mathbf{u}}}_{h}\right)\right|^{2}\left|\varepsilon\left(\overline{\mathbf{u}}_{h}\right)\right| d V .
\end{gathered}
$$


Proof. Inequality (5.18) follows from (5.15) and (3.3), while inequality (5.19) directly follows from (5.16). Computational details are given in [12].

Theorem 5.7. Suppose $\gamma=1$, let $\tau_{h}$ be a given mesh of $\Omega$, and let $\|\cdot\|$ be a norm of $V_{\mathrm{div}, h}$. There exist $\delta>0$ and $C>0$ such that if $\left\|\mathbf{u}_{h, 0}-\mathbf{u}_{h}\right\|<\delta$, then one has

$$
\left\|\mathbf{u}_{h}-\mathbf{u}_{h, k+1}\right\| \leq C\left\|\mathbf{u}_{h}-\mathbf{u}_{h, k}\right\|^{2},
$$

for all $k \geq 0$, and $\mathbf{u}_{h, k}$ is quadratically convergent to $\mathbf{u}_{h}$.

Proof. Owing to Theorem 5.3, there exists $\delta>0$ such that if $\left\|\mathbf{u}_{h, 0}-\mathbf{u}_{h}\right\|<\delta$, then $\| \mid \varepsilon\left(\mathbf{u}_{h, k}-\right.$ $\left.\mathbf{u}_{h}\right) \mid \|_{L^{\infty}} \rightarrow 0$ when $k \rightarrow \infty$. As a consequence, there exists $K_{1}$ such that $\left\|\left|\varepsilon\left(\mathbf{u}_{h, k}-\mathbf{u}_{h}\right)\right|\right\|_{L^{\infty}}<\delta$ for all $k>K_{1}$. By writing the Taylor expansion of $\mathcal{F}$ at point $\mathbf{u}_{h, k}$, there exists $\widehat{\mathbf{u}}_{h, k} \in V_{\mathrm{div}, h}$ such that

$$
\begin{array}{r}
\left\|\left|\varepsilon\left(\widehat{\mathbf{u}}_{h, k}-\mathbf{u}_{h, k}\right)\right|\right\|_{L^{\infty}}<\left\|\left|\varepsilon\left(\mathbf{u}_{h}-\mathbf{u}_{h, k}\right)\right|\right\|_{L^{\infty}}<\delta, \\
\mathcal{F}\left(\mathbf{u}_{h} ; \mathbf{v}_{h}\right)= \\
\mathcal{F}\left(\mathbf{u}_{h, k} ; \mathbf{v}_{h}\right)+\left\langle D \mathcal{F}\left(\mathbf{u}_{h, k} ; \mathbf{v}_{h}\right), \mathbf{u}_{h}-\mathbf{u}_{h, k}\right\rangle \\
+\frac{1}{2}\left\langle D^{2} \mathcal{F}\left(\widehat{\mathbf{u}}_{h, k} ; \mathbf{v}_{h}\right), \mathbf{u}_{h}-\mathbf{u}_{h, k}, \mathbf{u}_{h}-\mathbf{u}_{h, k}\right\rangle .
\end{array}
$$

Since $\mathbf{u}_{h}$ solves (4.4), then $\mathcal{F}\left(\mathbf{u}_{h} ; \mathbf{v}_{h}\right)=0$ in (5.22). By setting $\mathbf{v}_{h}=\mathbf{u}_{h}-\mathbf{u}_{h, k+1}$, we obtain, from (5.22) and (5.17),

$$
\left\langle D \mathcal{F}\left(\mathbf{u}_{h, k} ; \mathbf{u}_{h}-\mathbf{u}_{h, k+1}\right), \mathbf{u}_{h}-\mathbf{u}_{h, k+1}\right\rangle=-\frac{1}{2}\left\langle D^{2} \boldsymbol{F}\left(\widehat{\mathbf{u}}_{h, k} ; \mathbf{u}_{h}-\mathbf{u}_{h, k+1}\right), \mathbf{u}_{h}-\mathbf{u}_{h, k}, \mathbf{u}_{h}-\mathbf{u}_{h, k}\right\rangle .
$$

Thanks to (3.2), there exists $C_{0}=D_{1}\left(1+\left\|\varepsilon\left(\mathbf{u}_{h}\right)\right\|_{L^{\infty}}\right)^{1 / n-1}$ such that $0<C_{0} \leq \mu\left(\left|\varepsilon\left(\mathbf{u}_{h}\right)\right|\right)$. As a consequence, since $\left\|\left|\varepsilon\left(\mathbf{u}_{h, k}-\mathbf{u}_{h}\right)\right|\right\|_{L^{\infty}} \rightarrow 0$ and $\left\|\left|\varepsilon\left(\widehat{\mathbf{u}}_{h, k}-\mathbf{u}_{\mathbf{h}}\right)\right|\right\|_{L^{\infty}} \rightarrow 0$ when $k \rightarrow \infty$ and $\mu \in \mathcal{C}^{\infty}\left(\mathbb{R}_{+}^{*}\right)$, there exist $C_{1}, C_{2}>0$ and $K_{2} \geq K_{1}$ such that, for all $k>K_{2}$,

$$
\begin{gathered}
C_{1}<\mu\left(\left|\varepsilon\left(\mathbf{u}_{h, k}\right)\right|\right), \\
\left(8\left|\mu^{\prime}\left(\left|\varepsilon\left(\widehat{\mathbf{u}}_{h, k}\right)\right|\right)\right|+2\left|\mu^{\prime \prime}\left(\left|\varepsilon\left(\widehat{\mathbf{u}}_{h, k}\right)\right|\right)\right|\left|\varepsilon\left(\widehat{\mathbf{u}}_{h, k}\right)\right|\right)<C_{2} .
\end{gathered}
$$

By applying (5.18) with $\widetilde{\mathbf{u}}_{h}=\mathbf{u}_{h, k}$ and $\overline{\mathbf{u}}_{h}=\mathbf{u}_{h}-\mathbf{u}_{h, k+1}$ and (5.24), we obtain

$$
C_{1} \int_{\Omega}\left|\varepsilon\left(\mathbf{u}_{h}-\mathbf{u}_{h, k+1}\right)\right|^{2} d V \leq\left\langle D \mathcal{F}\left(\mathbf{u}_{h, k} ; \mathbf{u}_{h}-\mathbf{u}_{h, k+1}\right), \mathbf{u}_{h}-\mathbf{u}_{h, k+1}\right\rangle .
$$


By applying (5.19) with $\widetilde{\mathbf{u}}_{h}=\widehat{\mathbf{u}}_{h, k}, \overline{\mathbf{u}}_{h}=\mathbf{u}_{h}-\mathbf{u}_{h, k+1}$, and $\overline{\overline{\mathbf{u}}}_{h}=\mathbf{u}_{h}-\mathbf{u}_{h, k}$ and (5.25), we obtain:

$$
-\left\langle D^{2} \boldsymbol{F}\left(\widehat{\mathbf{u}}_{h, k} ; \mathbf{u}_{h}-\mathbf{u}_{h, k+1}\right), \mathbf{u}_{h}-\mathbf{u}_{h, k}, \mathbf{u}_{h}-\mathbf{u}_{h, k}\right\rangle \leq C_{2} \int_{\Omega}\left|\varepsilon\left(\mathbf{u}_{h}-\mathbf{u}_{h, k}\right)\right|^{2}\left|\varepsilon\left(\mathbf{u}_{h}-\mathbf{u}_{h, k+1}\right)\right| d V .
$$

By combining (5.23), (5.26), and (5.27), we obtain

$$
C_{1} \int_{\Omega}\left|\varepsilon\left(\mathbf{u}_{h}-\mathbf{u}_{h, k+1}\right)\right|^{2} d V \leq C_{2} \int_{\Omega}\left|\varepsilon\left(\mathbf{u}_{h}-\mathbf{u}_{h, k}\right)\right|^{2}\left|\varepsilon\left(\mathbf{u}_{h}-\mathbf{u}_{h, k+1}\right)\right| d V .
$$

By using Cauchy-Schwarz's inequality and the equivalence of norms, there exists $C_{3}>0$ such that

$$
\left\|\left|\varepsilon\left(\mathbf{u}_{h}-\mathbf{u}_{h, k+1}\right)\right|\right\|_{L^{2}}^{2} \leq C_{3}\left\|\left|\varepsilon\left(\mathbf{u}_{h}-\mathbf{u}_{h, k}\right)\right|\right\|_{L^{2}}^{2}\left\|\left|\varepsilon\left(\mathbf{u}_{h}-\mathbf{u}_{h, k+1}\right)\right|\right\|_{L^{2}} .
$$

Clearly, (5.20) follows from (5.29).

Remark 5.8. If $\Gamma_{R} \neq \emptyset$, the nonlinear Robin-Dirichlet condition can be handled in the same way as for the viscosity function. In that case, we modify the application $E$ by adding

$$
\sum_{i=1, d-1}\left(\int_{\Gamma_{R}} \alpha\left(\left|\tilde{\mathbf{u}}_{h}\right|\right)\left(\widetilde{\mathbf{w}}_{h} \cdot \mathbf{t}_{i}\right)\left(\mathbf{v}_{h} \cdot \mathbf{t}_{i}\right) d S+\gamma \int_{\Gamma_{R}} \frac{\alpha^{\prime}\left(\left|\tilde{\mathbf{u}}_{h}\right|\right)}{\left|\widetilde{\mathbf{u}}_{h}\right|}\left(\widetilde{\mathbf{u}}_{h} \cdot\left(\widetilde{\mathbf{w}}_{h}-\widetilde{\mathbf{u}}_{h}\right)\right)\left(\widetilde{\mathbf{u}}_{h} \cdot \mathbf{t}_{i}\right)\left(\mathbf{v}_{h} \cdot \mathbf{t}_{i}\right) d S\right)
$$

to the left-hand side of (5.2). Theorems 5.3 and 5.7 can be easily extended to this case.

\section{Numerical Results}

In this section, numerical experiences are performed in two dimensions $(d=2)$ to validate the results of Theorems 4.9, 5.3, and 5.7. An exact solution of the Stokes problem (2.1) in the square $\Omega=[0,1]^{2}$ is considered in the pure Dirichlet case, that is, $\partial \Omega=\Gamma_{D}$. Let

$$
\mathbf{u}(x, y)=\left(\begin{array}{c}
(x(1-x))^{\theta+1}(y(1-y))^{\theta}(1-2 y) \\
-(x(1-x))^{\theta}(y(1-y))^{\theta+1}(1-2 x)
\end{array}\right), \quad \theta \in[1,2]
$$

be a divergence-free velocity field that vanishes on the boundaries of $\Omega$. Let $p(x, y)=x y-$ $1 / 4$ be the pressure field such that $\int_{\Omega} p d V=0$. The right-hand side term $\mathbf{f}$ is deduced by computing (2.1). The parameters involving in Glen's law are $n=2, \tau_{0}=0.1$ bar and $A=$ $0.1 \mathrm{bar}^{-2} \mathrm{a}^{-1}$. Since $n=2$, the function $\mu$ defined by (2.2) is given explicitly, when $s>0$, by

$$
\mu(s)=\frac{\left(\sqrt{\left(2 A \tau_{0}\right)^{2}+8 \sqrt{2} A s}-2 A \tau_{0}\right)}{(4 \sqrt{2} A s)} .
$$


Numerical solutions are obtained after several successive approximations $\mathbf{u}_{h, k}$, as described in Section 5. Each $\mathbf{u}_{h, k}$ corresponds to a unique $p_{h, k}$. The algorithm is initialised by $\left(\mathbf{u}_{h, 0}, p_{h, 0}\right):=$ $(\mathbf{0}, 0)$. Each linearised problem is solved by using the finite element open source code Freefem++; see [24]. As spaces $V_{h}$ and $Q_{h}$, we opt for the combination $\left[\mathbb{P}_{1} / \text { Bulle }\right]^{d}-\mathbb{P}_{1}$ that satisfy the inf-sup condition (4.1) and the interpolation properties (4.26) and (4.27); see Remark 4.10. Six Delaunay unstructured regular meshes $\tau_{h}$ of the square $\Omega=[0,1]^{2}$ are generated with various resolutions $h$. Since the Dirichlet condition $\mathbf{u}=\mathbf{0}$ is applied on the whole boundary $\partial \Omega=\Gamma_{D}$, a penalisation term is added in the variational formulation to constrain the pressure average to be close to zero. For all norms $\|\cdot\|$, the error between $\mathbf{u}$ and $\mathbf{u}_{h, k}$ has two components:

$$
\left\|\mathbf{u}-\mathbf{u}_{h, k}\right\| \leq\left\|\mathbf{u}-\mathbf{u}_{h}\right\|+\left\|\mathbf{u}_{h}-\mathbf{u}_{h, k}\right\|
$$

where $\mathbf{u}_{h}$ is the exact solution of the nonlinear discrete problem. The convergence of the first component $\left\|\mathbf{u}-\mathbf{u}_{h}\right\|$ with respect to $h$ is the concern of Theorem 4.9, while the convergence of the second component $\left\|\mathbf{u}_{h}-\mathbf{u}_{h, k}\right\|$ with respect to $k$ is the concern of Theorems 5.3 and 5.7. Let $\bar{k}$ be an integer large enough such that $\mathbf{u}_{h}$ and $\mathbf{u}_{h, \bar{k}}$ can be confused, that is, such that $\left\|\mathbf{u}_{h}-\mathbf{u}_{h, \bar{k}}\right\| \ll\left\|\mathbf{u}-\mathbf{u}_{h}\right\|$. To check the convergence of the second component, we compute the following error:

$$
E_{k}^{u}=\frac{\left\|\nabla \mathbf{u}_{h, \bar{k}}-\nabla \mathbf{u}_{h, k}\right\|_{L^{r}}}{\|\nabla \mathbf{u}\|_{L^{r}}}
$$

where the norm $\|\cdot\|_{L^{r}}$ is evaluated by using the trapezoidal rule. For a fixed $h$, Theorem 5.3 states the linear convergence of $E_{k}^{u}$ that depends on constant (5.13) when $\gamma \in[0,1)$ and the quadratic convergence when $\gamma=1$. Three values of $\gamma$ are considered: $\gamma=0$ to test the fixed point algorithm, $\gamma=0.5$ to test the hybrid method, and $\gamma=1$ to test Newton's method. Figure 1 displays $E_{k}^{u}$ according to $k$ for each method: $\gamma \in\{0,0.5,1\}$, and for two different meshes. The recorded orders of convergence are consistent with Theorem 5.3: Newton's method $(\gamma=1)$ converges quadratically, the fixed point method and the hybrid method $(\gamma<1)$ converge linearly, and convergence is faster for bigger $\gamma$ and then smaller constant (5.13). Newton's method is especially very efficient: in our example, only 3 iterations are needed against 8 for the fixed point algorithm to obtain the same accuracy of the numerical solution. Figure 1 also shows that the convergence of $E_{k}^{u}$ with respect to $k$ is not affected by any mesh refinement, as noticed in [3]. Moreover, the addition of supplementary terms in the Stokes system does not increase significantly the computational time for solving the linear system with a direct method.

The estimate of Theorem 4.9 is now tested by computing the following errors:

$$
E_{h}^{u}=\frac{\left\|\nabla \mathbf{u}-\nabla \mathbf{u}_{h, \bar{k}}\right\|_{L^{r}}}{\|\nabla \mathbf{u}\|_{L^{r}}}, \quad E_{h}^{p}=\frac{\left\|p-p_{h, \bar{k}}\right\|_{L^{\kappa^{\prime}}}}{\|p\|_{L^{\kappa^{\prime}}}} .
$$

We can change the regularity of $\mathbf{u}$ by changing the parameter $\theta$ in (6.1) from 2 to 1.34. Indeed, if $\theta=2$, then $\mathbf{u} \in \mathcal{C}^{\infty}(\Omega)$, while if $\theta=1.34$, then $\mathbf{u} \notin\left[W^{2,2}(\Omega)\right]^{2}$, but $\mathbf{u} \in\left[W^{2, r}(\Omega)\right]^{2}$, where $r=3 / 2$. In any case $p \in \mathcal{C}^{\infty}(\Omega)$. Figure 2 displays $E_{h}^{u}$ and $E_{h}^{p}$ with respect to $h$ in both cases 


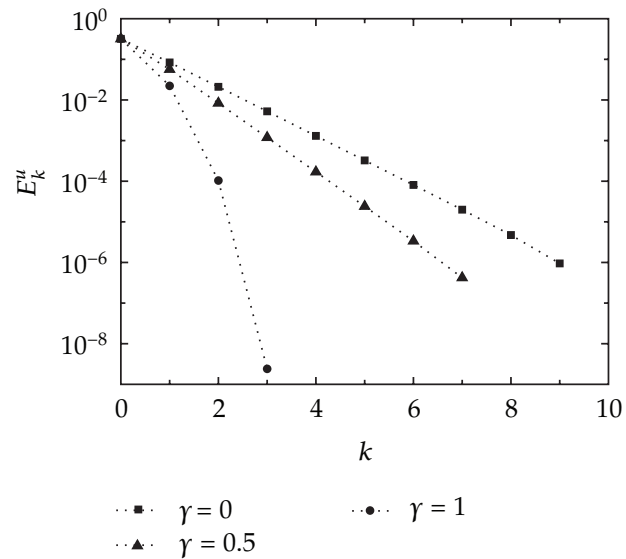

(a)

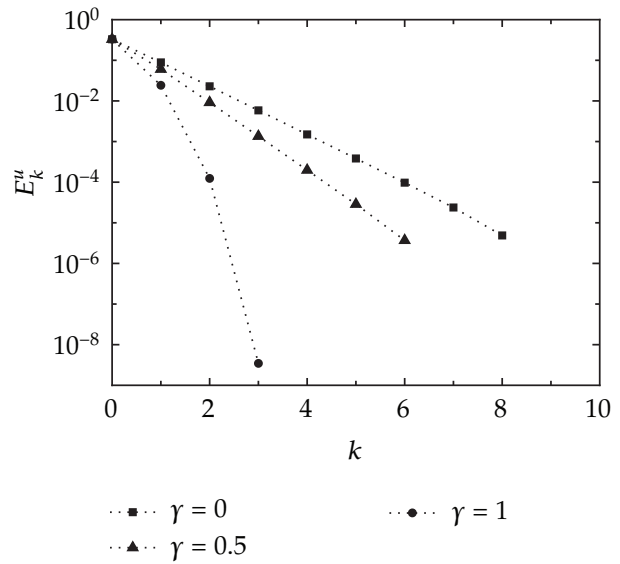

(b)

Figure 1: The error $E_{k}^{u}$ with respect to $k$ for each method (fixed point: $\gamma=0$, hybrid: $\gamma=0.5$, Newton: $\gamma=1$ ). The error $E_{k}^{u}$ obtained with the coarsest mesh is displayed on (a) and the error $E_{k}^{u}$ obtained with the finest mesh is displayed on (b).

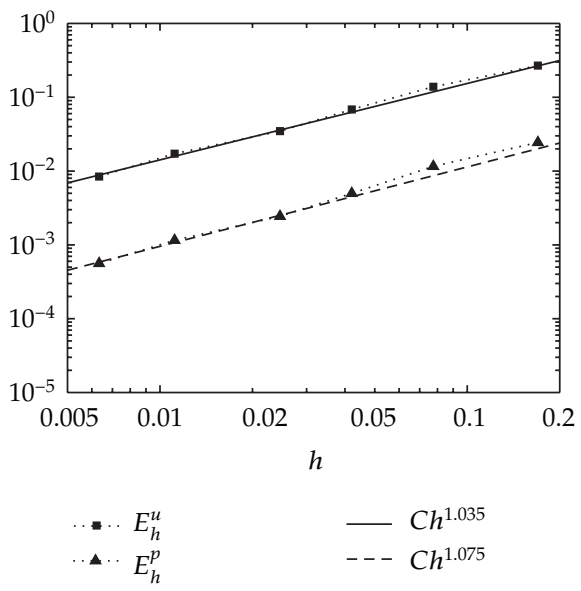

(a)

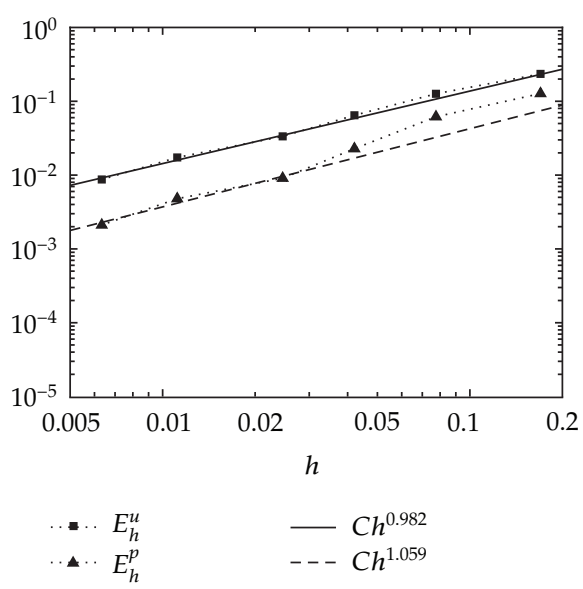

(b)

Figure 2: The errors $E_{h}^{u}$ and $E_{h}^{p}$ with respect to $h$ with $\theta=2$ (a) and with $\theta=1.34$ (b). Regression straight lines have been drawn from the last three points of each set of six recorded errors.

$\theta=2$ and $\theta=1.34$. The estimate (4.28) anticipates $E_{h}^{u}=\mathcal{O}(h), E_{h}^{p}=\mathcal{O}(h)$ if $\theta=2$, and $E_{h}^{u}=\mathcal{O}\left(h^{3 / 4}\right), E_{h}^{p}=\mathcal{O}\left(h^{1 / 2}\right)$ if $\theta=1.34$. In both cases, the observed order of convergence for $E_{h}^{u}$ and $E_{h}^{p}$ is close to one, which is greater or equal to the estimate. It suggests the nonoptimality of estimate (4.28) in the nonregular case, as noticed in [7] for a comparable problem.

\section{Conclusions and Perspectives}

We have proved the existence and the uniqueness of a weak solution of a nonlinear Stokes problem that describes the motion of glaciers. We have also proved the convergence 
of the finite element approximation and given a priori error estimates. New successive approximation algorithms have been proposed to solve the system nonlinearity and have been proved to be convergent. When implementing Newton's method, both theoretical and numerical studies have shown the efficiency of this method in comparison with the classical fixed point method.

Two extensions of our work should be investigated in future research. First, a posteriori estimates could be an aspect to be developed in order to implement an adaptive mesh procedure. Second, the presented Stokes model could benefit from recent improvements of the basal sliding description with Coulomb-type laws [10].

\section{Acknowledgments}

This work was funded by the Swiss National Science Foundation, Projects nos. 200021-119721 and PBELP2-133349. The authors thank H. Weaver for the English corrections.

\section{References}

[1] J. W. Glen, "The flow law of ice," IUGG/IAHS Symposium of Chamonix IAHS Publication, vol. 47, pp. 171-183, 1958.

[2] H. Blatter, "Velocity and stress fields in grounded glaciers: a simple algorithm for including deviatoric stress gradients," Journal of Glaciology, vol. 41, no. 138, pp. 333-344, 1995.

[3] J. Colinge and J. Rappaz, "A strongly nonlinear problem arising in glaciology," Mathematical Modelling and Numerical Analysis, vol. 33, no. 2, pp. 395-406, 1999.

[4] J. Baranger and K. Najib, "Analyse numérique des écoulements quasi-newtoniens dont la viscosité obéit à la loi puissance ou la loi de carreau," Numerische Mathematik, vol. 58, no. 1, pp. 35-49, 1990.

[5] J. W. Barrett and W. B. Liu, "Quasi-norm error bounds for the finite element approximation of a nonNewtonian flow," Numerische Mathematik, vol. 68, no. 4, pp. 437-456, 1994.

[6] S. S. Chow, G. F. Carey, and M. L. Anderson, "Finite element approximations of a glaciology problem," Mathematical Modelling and Numerical Analysis, vol. 38, no. 5, pp. 741-756, 2004.

[7] R. Glowinski and J. Rappaz, "Approximation of a nonlinear elliptic problem arising in a non-Newtonian fluid flow model in glaciology," Mathematical Modelling and Numerical Analysis, vol. 37, no. 1, pp. 175-186, 2003.

[8] J. Rappaz and A. Reist, "Mathematical and numerical analysis of a three-dimensional fluid flow model in glaciology," Mathematical Models and Methods in Applied Sciences, vol. 15, no. 1, pp. 37-52, 2005.

[9] A. Reist, Mathematical analysis and numerical simulation of the motion of a glacier, Ph.D. thesis, EPF Lausanne, Lausanne, Switzerland, 2005.

[10] C. Schoof, "Coulomb friction and other sliding laws in a higher-order glacier flow model," Mathematical Models and Methods in Applied Sciences, vol. 20, no. 1, pp. 157-189, 2010.

[11] R. Greve and H. Blatter, Dynamics of Ice Sheets and Glaciers, Springer, New York, NY, USA, 2009.

[12] G. Jouvet, Modélisation, analyse mathématique et simulation numérique de la dynamique des glaciers, Ph.D. thesis, EPF Lausanne, Lausanne, Switzerland, 2010.

[13] K. Hutter, Theoretical Glaciology, Reidel, 1983.

[14] G. Jouvet, M. Huss, H. Blatter, M. Picasso, and J. Rappaz, "Numerical simulation of Rhonegletscher from 1874 to 2100," Journal of Computational Physics, vol. 228, no. 17, pp. 6426-6439, 2009.

[15] A. Ern and J. L. Guermond, Theory and Practice of Finite Elements, Springer, New York, NY, USA, 2004.

[16] V. Girault and P. A. Raviart, Finite Element Methods for Navier-Stokes Equations: Theory and Algorithms, Springer Series in Computational Mathematics, Springer, New York, NY, USA, 1986.

[17] A. Quarteroni and A. Valli, Numerical Approximation of Partial Differential Equations, Springer, Berlin, Germany, 1994.

[18] W. Pompe, "Korn's first inequality with variable coefficients and its generalization," Commentationes Mathematicae Universitatis Carolinae, vol. 44, no. 1, pp. 57-70, 2003.

[19] H. Brezis, Analyse Fonctionnelle, Théorie et Applications, Dunod, 1999. 
[20] J. Howell and N. Walkington, "Inf-sup conditions for twofold saddle point problems," Numerische Mathematik, vol. 118, pp. 663-693, 2011.

[21] C. Amrouche and V. Girault, "Decomposition of vector spaces and application to the Stokes problem in arbitrary dimension," Czechoslovak Mathematical Journal, vol. 44, no. 1, pp. 109-140, 1994.

[22] A. Quarteroni and A. Valli, Domain Decomposition Methods for Partial Differential Equations, Oxford University Press, Oxford, UK, 1999.

[23] D. N. Arnold, F. Brezzi, and M. Fortin, "A stable finite element for the stokes equations," Calcolo, vol. 21, no. 4, pp. 337-344, 1984.

[24] F. Hecht, Freefem++, 2010. 


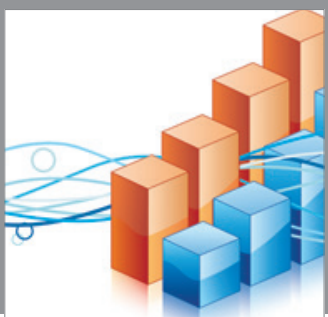

Advances in

Operations Research

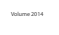

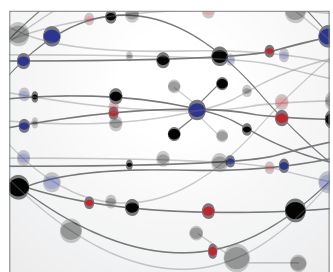

\section{The Scientific} World Journal
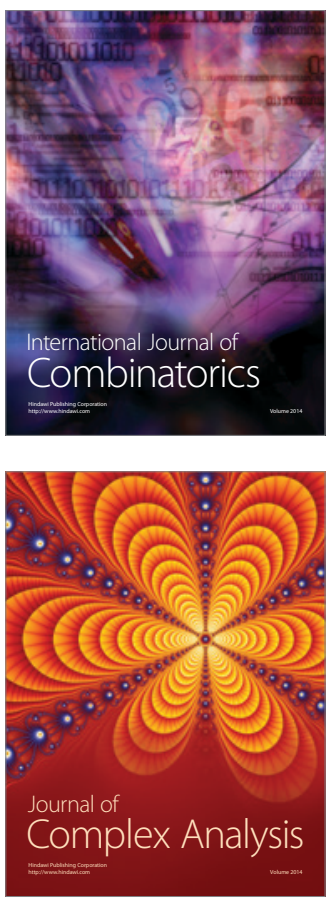

International Journal of

Mathematics and

Mathematical

Sciences
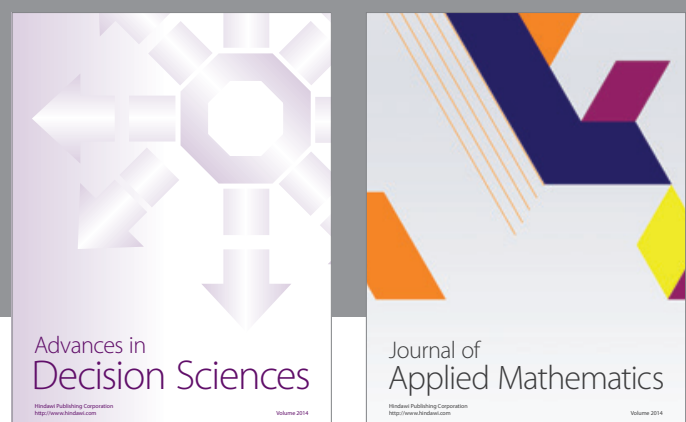

Journal of

Applied Mathematics
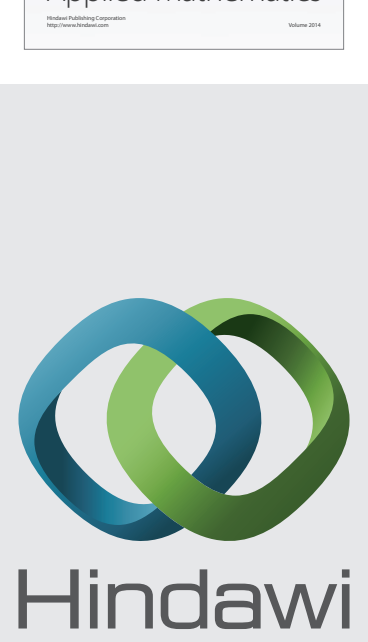

Submit your manuscripts at http://www.hindawi.com
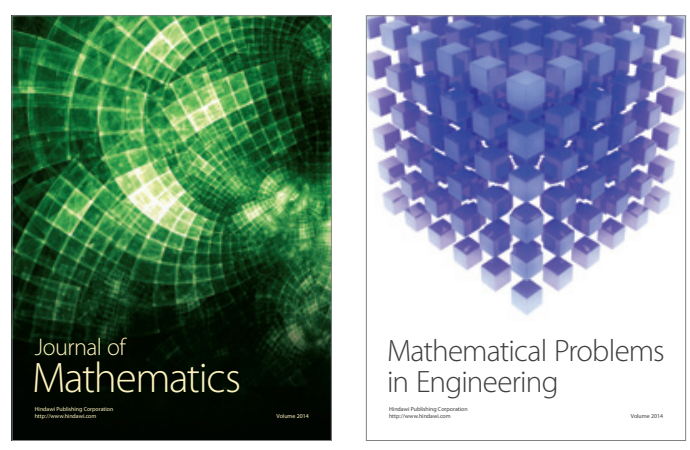

Mathematical Problems in Engineering
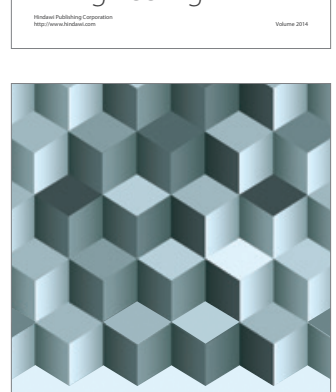

Journal of

Function Spaces
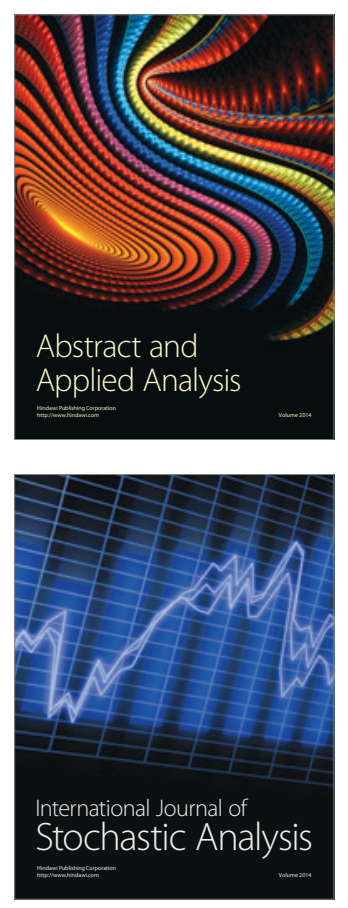

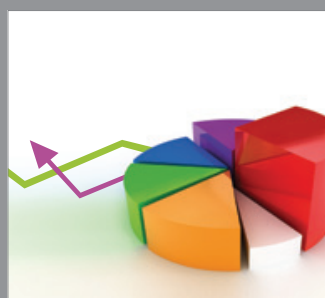

ournal of

Probability and Statistics

Promensencen
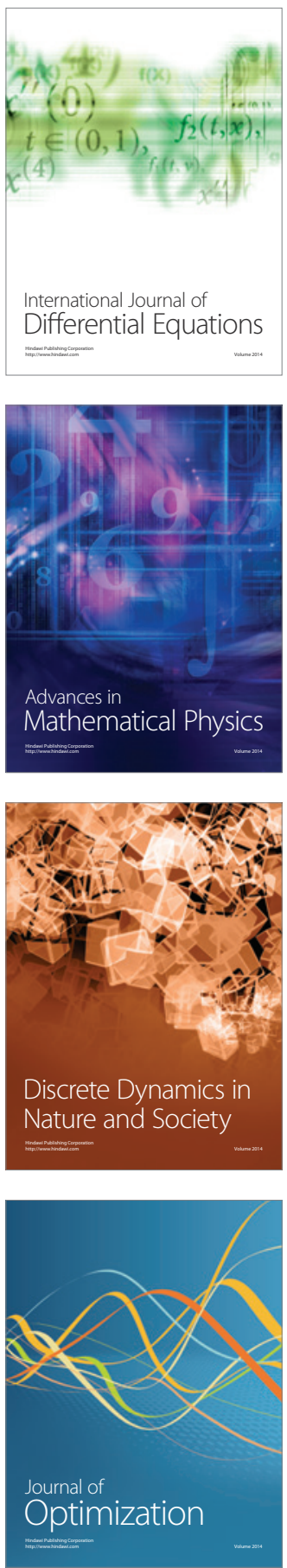